\title{
Geochemical Characteristics of Cenozoic Jining Basalts of the Western North China Craton: Evidence for the Role of the Lower Crust, Lithosphere, and Asthenosphere in Petrogenesis
}

\author{
Kung-Suan $\mathrm{Ho}^{1, *}$, Yan Liu ${ }^{2}$, Ju-Chin Chen ${ }^{3}$, Chen-Feng You ${ }^{4,5}$, and Huai-Jen Yang ${ }^{4}$ \\ ${ }^{1}$ Department of Geology, National Museum of Natural Science, Taichung, Taiwan, ROC \\ ${ }^{2}$ Institute of Geology, Chinese Academy of Geological Sciences, Beijing, China \\ ${ }^{3}$ Institute of Oceanography, National Taiwan University, Taipei, Taiwan, ROC \\ ${ }^{4}$ Department of Earth Sciences, National Cheng Kung University, Tainan, Taiwan, ROC \\ ${ }^{5}$ Earth Dynamic System Research Center, National Cheng Kung University, Tainan, Taiwan, ROC
}

Received 11 December 2009, accepted 28 June 2010

\begin{abstract}
The Jining volcanic field located in the southern margin of the Mongolian plateau and the western North China Block consists of four rock types: quartz tholeiite, olivine tholeiite, alkali olivine basalt and basanite. These rocks have a wide range of $\mathrm{K}$-Ar ages from $\sim 36$ to $<0.2 \mathrm{Ma}$. The early volcanism was voluminous and dominated by flood-type fissure eruptions of tholeiites, whereas the later phase was represented by sparse eruptions of basanitic lavas. Thirty-six samples analyzed in this study show a wide range in $\mathrm{SiO}_{2}$ contents from $44 \% \sim 54 \%$. They all are sodium-rich and high-Ti basalts that, however, show marked isotopic variations between two end-members: (1) tholeiites that have higher ${ }^{87} \mathrm{Sr} /{ }^{86} \mathrm{Sr}$ of $0.7048 \sim 0.7052$, and lower $\varepsilon \mathrm{Nd}$ of -0.8 to -2.4 and $\mathrm{Pb}$ isotope ratios $\left({ }^{206} \mathrm{~Pb} /{ }^{204} \mathrm{~Pb}\right.$ of $16.9 \sim 17.2,{ }^{207} \mathrm{~Pb} /{ }^{204} \mathrm{~Pb}$ of $15.3 \sim 15.4$ and ${ }^{208} \mathrm{~Pb} /{ }^{204} \mathrm{~Pb}$ of $\left.37.1 \sim 37.7\right)$; and (2) basanites that have lower ${ }^{87} \mathrm{Sr} /{ }^{86} \mathrm{Sr}$ of $0.7035 \sim 0.7044$, and higher $\varepsilon \mathrm{Nd}$ of +1.3 to +4.9 and $\mathrm{Pb}$ isotope ratios $\left({ }^{206} \mathrm{~Pb} /{ }^{204} \mathrm{~Pb}\right.$ of $17.7 \sim 18.0,{ }^{207} \mathrm{~Pb} /{ }^{204} \mathrm{~Pb}$ of $15.4 \sim 15.5$ and ${ }^{208} \mathrm{~Pb} /{ }^{204} \mathrm{~Pb}$ of $37.8 \sim 38.2$ ). Alkali olivine basalt that occurs as a subordinate rock type is geochemically similar to the basanites, but isotopically similar to the tholeiites, characterized by the highest ${ }^{87} \mathrm{Sr} /{ }^{86} \mathrm{Sr}$ ratio among the three basaltic suites, coupled with a low $\mathrm{Nb} / \mathrm{U}$ value $(\sim 33)$.

In $\mathrm{Sr}-\mathrm{Nd}-\mathrm{Pb}$ isotopic plots, the tholeiites extend toward the EM1 (i.e., enriched mantle type 1) component, whereas the basanites trend toward the Indian Ocean mid-ocean ridge basalt (MORB) field. Adopting the 'plum-pudding' model by Morris and Hart (1983), we suggest that the Oligocene tholeiites were generated by high degree melting of an ascended asthenospheric mantle that was contaminated with a large amount of EM1-type continental lithospheric material during the early Cenozoic. On the other hand, the late Tertiary and Quaternary basanites may have originated predominantly from a depleted asthenosphere component with small but variable degrees of contribution by the continental lithospheric mantle. The generation of alkali olivine basalt requires addition of a two-pyroxene granulite component, thus we suggest it was derived from a basanitic parental magma that underwent minor amounts of lower crustal contamination during ascent to the surface.
\end{abstract}

Key words: K-Ar dating, Sr-Nd-Pb isotope, Petrogenesis, Cenozoic Jining basalts, Western North China Craton

Citation: Ho, K. S., Y. Liu, J. C. Chen, C. F. You, and H. J. Yang, 2011: Geochemical characteristics of Cenozoic Jining basalts of the western North China Craton: Evidence for the role of the lower crust, lithosphere, and asthenosphere in petrogenesis. Terr. Atmos. Ocean. Sci., 22, 15-40, doi: 10.3319/ TAO.2010.06.28.01(TT)

\section{INTRODUCTION}

During the past few decades, many studies have been conducted to identify processes involved in the generation of continental basaltic magmatism. The geochemical charac-

\footnotetext{
* Corresponding author

E-mail:ksho@mail.nmns.edu.tw
}

teristics, especially the isotopic ratios, of the volcanic rocks in different sub-provinces are somewhat variable and complex. In general, the trace-element and isotopic heterogeneity exhibited by continental basalts is thought to reflect the compositional diversity of the mantle materials from which they were derived. They may reflect the intrinsic heterogeneity of mantle sources, asthenosphere-lithospheric mantle 
interactions (including lithospheric delamination), oceanic crust-lithospheric mantle interaction, or contamination of mantle-derived melts by crustal materials (e.g., Glazner and Farmer 1992; Chung et al. 1994; Zhang et al. 2009).

Cenozoic intraplate basalts are widely distributed in eastern China. Geochemical and isotopic studies showed that basaltic rocks from this region are highly heterogeneous, and that distinct mantle source regions must have been involved in the generation of these magmas (Song et al. 1990). Chen et al. (2007) concluded that the isotopic variability of the Northeast China volcanic rocks is encompassed by two end members: FOZO (Focal zone) and LoMu (low ' $\mu$ '). The general considerations of mantle sources for these basalts have a depleted mid-ocean ridge basalt (MORB) mantle (DMM)-enriched mantle of type 1 (EM1) array for North/Northeast China and a DMM-EM2 array for South China (e.g., Xie et al. 1989; Tu et al. 1992). According to the suggestions of most workers, the EM1/EM2 component is the subcontinental lithospheric mantle, whereas the DMM component is the asthenospheric mantle that has invaded the lithospheric mantle as a result of thermotectonic processes (e.g., Basu et al. 1991; Chung et al. 1994).

The continental lithosphere usually plays an important role by forming a barrier to continued upwelling of the asthenospheric mantle. Therefore, except for ancient subduction processes, lithospheric contamination of the asthenospheric mantle can also occur through lithospheric delamination (McKenzie and O’Nions 1995). In addition, lithospheric thinning in eastern China was also widely discussed in the last two decades. It is well established that tectonothermal events resulted in replacement of the old, cold, thick, depleted lithospheric mantle by a young, hot, thin, and fertile lithospheric mantle accompanied by lithospheric thinning in the eastern North China Craton (NCC, Griffin et al. 1998). In contrast, the lithospheric mantle beneath the Western Block, NCC was progressively thinned and may consist mainly of old lithospheric relicts after thermo-mechanical erosion (Xu 2001). The most recent studies of Fanhsi peridotite xenoliths revealed isotopic and chemical heterogeneities, which reflect the refertilization of the ancient refractory lithospheric mantle by the addition of asthenospheric melts (Tang et al. 2008).

Previous work on Cenozoic continental volcanism in the western NCC (i.e., west of the Daxinganling-Taihangshan gravity lineament, DTGL, Fig. 1a) mainly concentrated in the Fanhsi, Hannuoba, and Datong regions (e.g., Zhi et al. 1990; Xu et al. 2005; Tang et al. 2006); however, this article focuses on the basaltic rocks of the Jining volcanic field (JVF). Comparison with lavas from adjacent volcanic fields of Fanhsi, Hannuoba, and Datong showed that they had shorter periods of volcanic activities, lasting for 17, 22, and $<1 \mathrm{Ma}$, respectively, and had limited ranges of isotopic compositions. It was demonstrated in this study that the major- and trace-element concentrations and $\mathrm{Sr}-\mathrm{Nd}-\mathrm{Pb}$ isotopic compositions of the Jining basalts have changed substantially throughout $36 \mathrm{Ma}$ of volcanic history. Therefore, the Cenozoic basaltic rocks from the JVF may provide important information to establish the mantle evolution underneath the western NCC.

Few Jining basalts have been studied geochronologically (Luo and Chen 1990; Liu et al. 1992b), but no detailed geochemical information, especially on $\mathrm{Sr}-\mathrm{Nd}-\mathrm{Pb}$ isotope ratio analyses, is available. In the present study, the first issue concerns the eruption age of large-volume magmatism and the second issue concerns the relative roles of the lower crust, continental lithosphere, and asthenosphere as sources for the magmas. Hence, systematic radiometric ages, and geochemical and $\mathrm{Sr}-\mathrm{Nd}-\mathrm{Pb}$ isotopic analyses are reported for the Jining basalts. Our new results on basaltic rocks allowed us to examine spatial and temporal variations in the eruptive ages and chemistry of Cenozoic volcanism in this region; accordingly, we were able to determine the general trend of their compositional evolution, and understand the nature of the mantle sources and melt-generating processes.

\section{GEOLOGICAL SETTING AND FIELD DESCRIP- TION}

The JVF lies at the juncture of Shanxi Province and Inner Mongolia Autonomous Region, China. It is one of the three main areas, along with the Abaga and Chifeng volcanic fields, of Cenozoic intraplate basaltic activities in Inner Mongolia (Fig. 1). The lavas of the JVF form an extensive lava platform with exposure of more than $5000 \mathrm{~km}^{2}$ in the southern part of the Mongolian Plateau. Its areal extent is smaller than that of the Abaga volcanic field (> $10000 \mathrm{~km}^{2}$, Liu et al. 1992b) but larger than that of the Chifeng volcanic field ( $3000 \mathrm{~km}^{2}$, Han et al. 1999).

The NCC is one of the oldest continental nuclei in the world (Liu et al. 1992a; Zheng et al. 2004). Based on the lithological, geochemical, and metamorphic P-T path data of the basement rocks, the basement of NCC can be divided into three regions: the Western Block, Central Zone, and Eastern Block (Zhao et al. 2001). The JVF lies in the Western Block, which is a stable platform composed of Late Archean to Paleoproterozoic metasedimentary rocks unconformably overlying an Archean basement (Zhao et al. 2001) and is characterized by having crustal and lithospheric thicknesses of more than 40 and $100 \mathrm{~km}$, respectively (Ma 1989). Cenozoic volcanic fields within or near the Western Block, in addition to the JVF, include the Fanhsi (including the Fanhsi and Yinxian in northern Shanxi Province and Yangyuan in western Hebei Province), Hannuoba, and Datong areas, all of which are located west of the N-S trending Daxinganling-Taihangshan gravity lineament (Fig. 1). In addition, the JVF is located between the Yinchuan-Hetao rift and Shanxi-Shaanxi rift (Fig. 1). Both rift systems de- 
veloped after the Indo-Asia collision (ca. $50 \sim 70 \mathrm{Ma}$ ) and are still active today (Zhang et al. 1998d).

The JVF is mainly built of lava flows and small deposits of pyroclastic rocks, cinders, scoria, and tuff. Widespread Cenozoic basaltic volcanism in general has two occurrences: one of lava flow and the other of a volcanic cone. The former erupted along major extensional fissures and is usually composed of tholeiitic rocks, whereas the latter is predominantly small-volume alkaline basalts and is situated in the northern part of the JVF, near Qahar Youyi Houqi City. On the basis of drill-core or field-profile stratigraphies, several eruptive episodes can be recognized. For instance, the basaltic profile at $30 \mathrm{~km}$ northeast of Jining City consists of five thick lava flows, each typically 15 $30 \mathrm{~m}$ thick, that are separated by thin layers of sedimentary formations. Field investigations showed that the maximum thickness of the Jining basaltic flows can reach $250 \mathrm{~m}$ (Liu et al. 1992b).

(a)

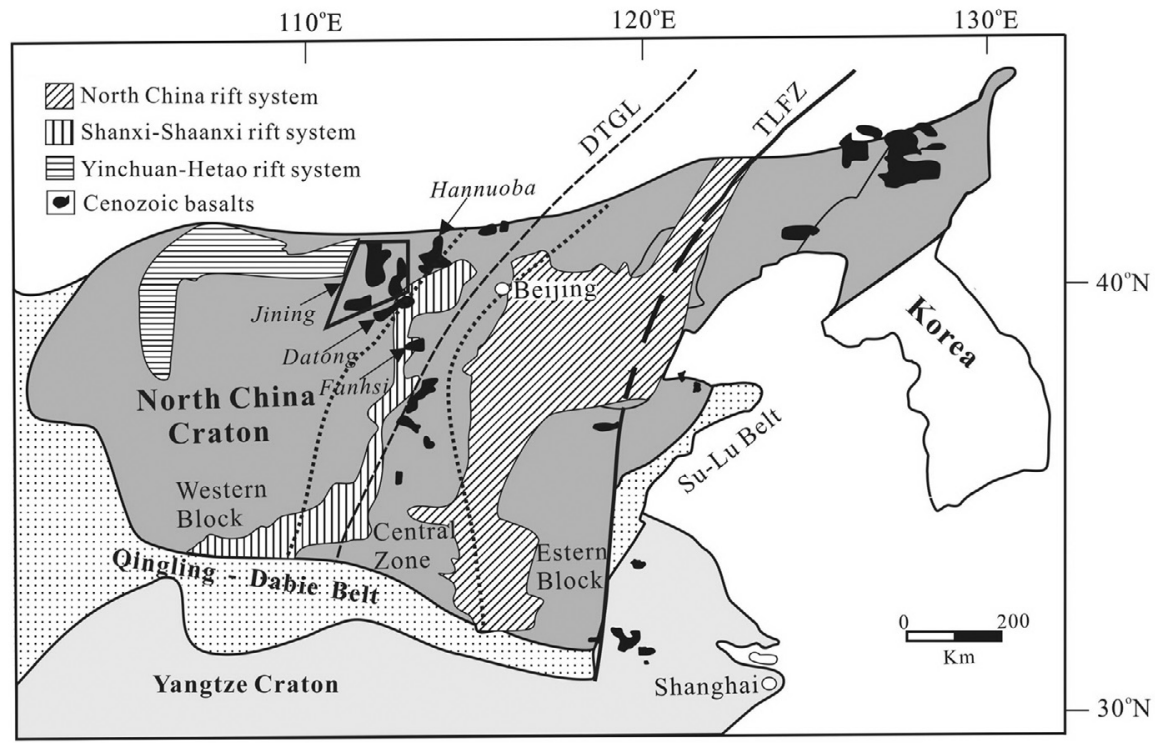

(b)

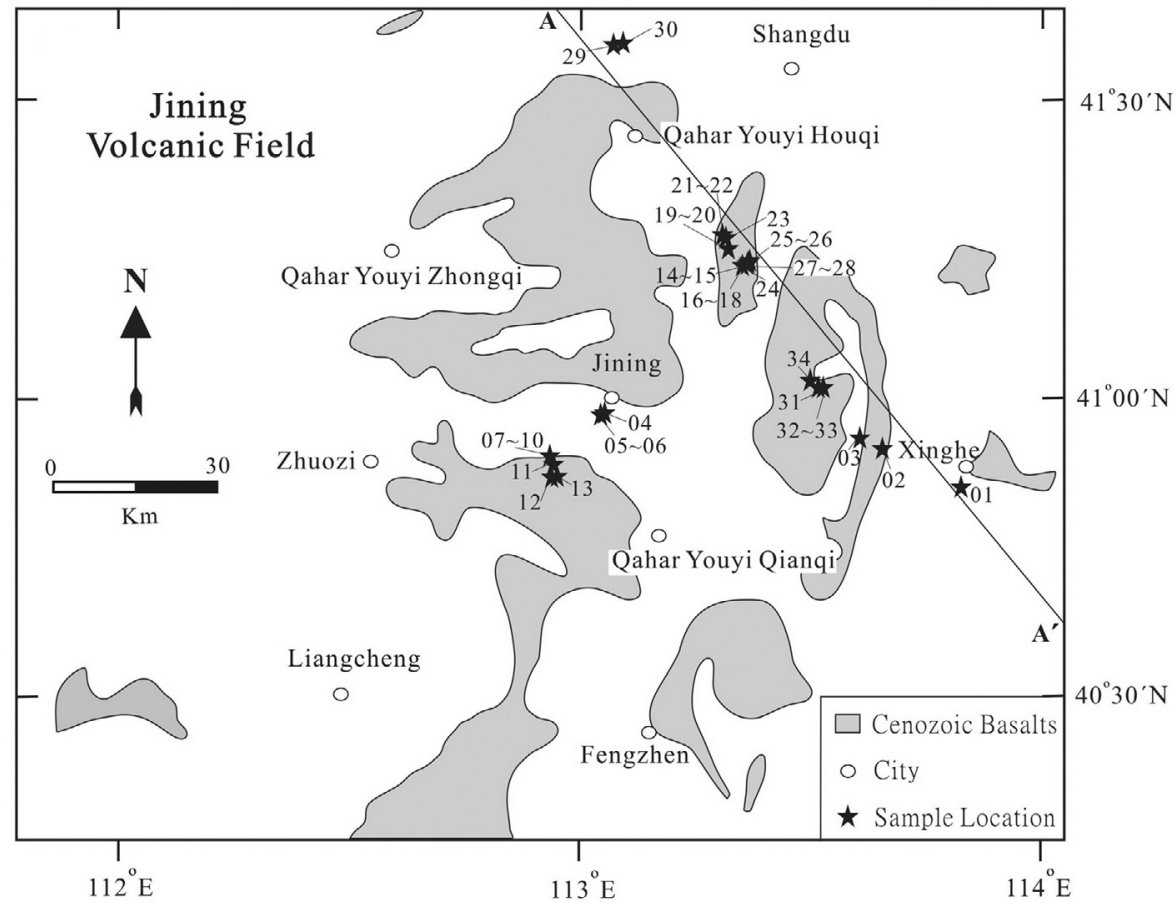

Fig. 1. (a) Simplified tectonic map showing the major tectonic units of the North China Craton (NCC; after Zhao et al. 2001). Cenozoic basaltic rocks in the western NCC can be subdivided into four volcanic fields: Jining, Hannuoba, Fanhsi, and Datong. TLFZ, Tan-Lu Fault Zone; DTGL, Daxinganling-Taihangshan gravity lineament. A boxed area is shown in (b). (b) The sketch map shows the distribution of Jining lavas and sample locations (modified from the Bureau of Geology and Mineral Resources of Inner Mongolia Autonomous Region 1991). Some samples were collected from the basalt section and were marked with linked number. The cross-section line for Fig. 13 is labeled as A-A'. 


\section{ANALYTICAL METHODS}

Fifteen whole-rock samples, after the removal of altered surfaces, were crushed into fragments of $20 \sim 80$ mesh in size and $20.50 \sim 142.75 \mathrm{mg}$ in weight for K-Ar dating. $\mathrm{K}$ abundances (wt \%) were determined using an HG-3 flamephotometer. Radiogenic ${ }^{40} \mathrm{Ar}$ was measured using the isotope dilution method and MM120 mass spectrometers at the State Key Laboratory of Earthquake Dynamics, Institute of Geology, China Earthquake Administration, Beijing, China. The decay constants and conversion factors used were those recommended by Steiger and Jäger (1977).

Thirty-six samples were analyzed for major and trace element abundances. All elements except Si were determined using solutions prepared by dissolving $0.5 \mathrm{~g}$ of rock powder in a mixture of ultrapure $\mathrm{HF}$ and $\mathrm{HNO}_{3}$ in Teflon beakers. The $\mathrm{Si}$ solution was prepared by $\mathrm{NaOH}$ fusion of $0.05 \mathrm{~g}$ of rock powder in nickel crucibles followed by water leaching and $\mathrm{HCl}$ acidification. Major oxides were analyzed using Perkin-Elmer Optima 2000DV inductively coupled plasma-atomic emission spectrometry (ICP-AES) at the National Museum of Natural Science, Taichung, Taiwan. Trace element abundances were obtained on an Agilent 7500ce ICP-mass spectrometer (MS) at the Department of Earth Sciences, National Cheng Kung University (DES/NCKU), Tainan, Taiwan. Calibration curves were constructed using US Geological Survey rock standards AGV-1, BCR-1, W-1, and G-2, and Geological Survey of Japan rock standard JB1 . The precision was estimated to be around $\pm 2 \%$ for the ICP-AES method and better than $\pm 5 \%$ for all ICP-MS analyses. Details of the analytical techniques were discussed by Ho et al. (2003).

Nineteen whole-rock samples were selected for $\mathrm{Sr}-\mathrm{Nd}$ isotopic analyses. The $\mathrm{Sr}$ and $\mathrm{Nd}$ fractions were separated by ion exchange chromatography, and isotopic compositions were measured using a MAT 262 mass spectrometer at the DES/NCKU. Procedural blanks for $\mathrm{Sr}$ and $\mathrm{Nd}$ were $<200 \mathrm{pg}$. Nd isotopic ratios were normalized to ${ }^{146} \mathrm{Nd} /{ }^{144} \mathrm{Nd}$ $=0.7219$ and are reported relative to 0.511855 for the $\mathrm{La}$ Jolla standard. Sr isotopic ratios were normalized to ${ }^{86} \mathrm{Sr} /{ }^{88} \mathrm{Sr}$ $=0.1194$ and are relative to 0.710240 for the SRM987 standard. The precisions of the $\mathrm{Nd}$ and $\mathrm{Sr}$ isotopic compositions were both better than $\pm 0.000010(2 \sigma)$.

$\mathrm{The} \mathrm{Pb}$-isotope data for 16 representative samples were obtained by a different laboratory. Isotopic ratios of six samples were measured on a Finnigan MAT-262 thermal ionization mass spectrometer (TIMS) at the Laboratory for Radiogenic Isotope Geochemistry, Institute of Geology and Geophysics, Chinese Academy of Sciences, Beijing, China. About $100 \mathrm{mg}$ of whole-rock powder of each sample was completely decomposed in a mixture of $\mathrm{HF}-\mathrm{HNO}_{3}$ for the $\mathrm{Pb}$ isotopic analysis. $\mathrm{Pb}$ was separated in Teflon ${ }^{\circledR}$ columns containing $\sim 80 \mu \mathrm{l}$ AG1-X8 of $100 \sim 200$ mesh and employing $\mathrm{HBr}-\mathrm{HCl}$ washing and elution procedures. The proce- dural blank was $<100 \mathrm{pg}$ for $\mathrm{Pb}$. For measuring the isotopic ratios, $\mathrm{Pb}$ was loaded with a mixture of Si-gel and $\mathrm{H}_{3} \mathrm{PO}_{4}$ onto a single-Re filament and measured at around $1300^{\circ} \mathrm{C}$. The NBS $981 \mathrm{~Pb}$ standard was used to determine thermal fractionation, and the measured isotopic ratios of samples were corrected with a value of $0.1 \%$ per atomic mass unit. Technical details of the chemical separation and measurement are described in Chen et al. (2000). In addition, Pbisotope analyses of ten samples were carried out on a highresolution multiple collector (MC)-ICP-MS (Thermo-Fisher Neptune) at the Earth Dynamic System Research Center, NCKU. Approximately $100 \mathrm{mg}$ of sample rock powder was loaded into a PFA beaker with $6.0 \mathrm{ml}$ of $65 \% \mathrm{HNO}_{3}$ and $4.0 \mathrm{ml}$ of $48 \% \mathrm{HF}$, and dissolved on a hotplate for 12 hours at $100^{\circ} \mathrm{C}$. A digested sample was dried down on a hotplate overnight at $100^{\circ} \mathrm{C}$ and was then taken up in $3 \mathrm{~N} \mathrm{HNO}_{3}$ with complete dissolution ensured by ultrasonication $(20 \mathrm{~min})$. Before the sample was loaded on a 300- $\mu$ l column of $\mathrm{Pb}$ spec $50 \sim 100$-mesh resin, the column was washed with two cycles of 18 mega ohm water/6 N HCl, and conditioned with 18 mega ohm water followed by $3 \mathrm{~N} \mathrm{HNO}_{3}$. Finally, the sample solution was centrifuged at $15000 \mathrm{rpm}$ for $3 \mathrm{~min}$, and the supernatant was loaded onto the column. Sr, Hf, and the REEs were washed from the column with $3 \mathrm{~N} \mathrm{HNO}_{3}$, after which $\mathrm{Pb}$ was eluted in $6 \mathrm{~N} \mathrm{HCl}$. Based on repeated analysis of NBS $981 \mathrm{~Pb}$ standards, the average values and $2 \sigma$ external precisions for $\mathrm{Pb}$ isotope analyses were 16.9418 $\pm 0.0042,15.4949 \pm 0.0053$, and $36.7194 \pm 0.0127$ for ${ }^{206} \mathrm{~Pb} /{ }^{204} \mathrm{~Pb},{ }^{207} \mathrm{~Pb} /{ }^{204} \mathrm{~Pb}$, and ${ }^{208} \mathrm{~Pb} /{ }^{204} \mathrm{~Pb}$, respectively.

\section{SAMPLING AND PETROGRAPHY}

The distribution of Cenozoic volcanic rocks in Jining and the sampling locations involved in the present study are shown in Fig. 1. Most samples were collected from quarries and road cuts; therefore, the analyzed samples were generally fresh in thin sections, except that olivine phenocrysts in some samples were partially altered or pseudomorphed by iddingsite.

Basaltic rocks were gray and compact, and lesser samples were vesicular. The rock texture of the basalts ranged from aphyric to porphyritic, although the former was rather uncommon and was confined to the basanites. The common phenocryst phases were plagioclase, augite, and olivine, with only tholeiitic rocks having phenocrystal Fe-Ti oxide minerals. The groundmass consisted of plagioclase, clinopyroxene, and olivine, with intergranular Fe-Ti oxide minerals or interstitial glass. In the study area, xenocrysts and xenoliths were not found in the basaltic rocks.

\section{K-AR AGES OF THE JINING BASALTS}

Previously, K-Ar dating data of Jining basaltic rocks were published by Luo and Chen (1990) and Liu et al. 
(1992b). Those analyzed samples were mainly collected from Qahar Youyi Houqi and Fenzhen by surface sampling and near Jining City by drilling cores. The deepest sample core $\left(\mathrm{JG}_{55}\right)$ was from the Jining area with a depth of $83.87 \mathrm{~m}$. Published data showed that the timing of volcanic activities were from 28.0 to 19.0 Ma in Qahar Youyi Houqi and from 14.3 to 10.2 Ma near Jining City. However, there were significant differences in $\mathrm{K}$-Ar dating results in Fenzhen basalts. A K-Ar dating study on Fenzhen lava by Liu et al. (1992b) yielded a consensus of ages clustering closely around $8 \mathrm{Ma}$. On the other hand, K-Ar dating data analyzed by Luo and Chen (1990) showed old ages of 16.3 14.2 Ma. In order to determine the times of volcanic eruptions in different areas and also to understand the evolutionary history of the basaltic magma, 15 fresh samples encompassing the full range of chemical diversity were selected for the K-Ar dating analysis, and the analytical results are given in Table 1.

We found that the oldest basaltic sample in the JVF had an age of $36.4 \pm 0.8 \mathrm{Ma}$, and the youngest age for a basanitic sample was $0.11 \pm 0.01 \mathrm{Ma}$. Published data by Luo and Chen (1990) and Liu et al. (1992b) showed that Cenozoic volcanism began in the Middle Miocene ( 28 Ma); however, new $\mathrm{K}-\mathrm{Ar}$ age data in this study suggested that it may have begun as early as the Oligocene $(\sim 36 \mathrm{Ma})$ and large-scale Cenozoic magmatic activity yielded ages of 36 $\sim 33 \mathrm{Ma}$, corresponding to the early Oligocene (Table 1). In addition, Quaternary volcanism in the NCC was rare and sparse. They included Datong volcanoes $(0.88 \sim 0.23 \mathrm{Ma}$, Wang et al. 1989; Chen et al. 1992; Ho, unpublished data) and rare alkali lavas in the Wulidashan, Shandong (0.75 Ma) and Nushan, Anhui (0.73 0.55 Ma, Liu et al. 1992b). No Quaternary basalt had ever been reported in the JVF before. In this study, two Quaternary volcanic rocks were determined to belong to basanite samples, which are volcanic cones, lie about $18 \mathrm{~km}$ to the NNW of Qahar Youyi Houqi City (Fig. 1).

\section{BULK CHEMISTRY}

\subsection{Major Elements}

The abundance of major elements of the representative basaltic rocks is listed in Table 2. Based on CIPW normative calculations and the Ne-Ol-Di-Hy-Qz tetrahedron of Yoder and Tilley (1962) as well as the classification scheme of alkali basalts proposed by Chih (1988), all volcanic

Table 1. K-Ar ages of whole rock of basalts from the Jining volcanic field, Inner Mongolia.

\begin{tabular}{|c|c|c|c|c|c|c|}
\hline Sample No. & Rock Type & Weight Analyzed (mg) & $\mathbf{K}(\%)$ & ${ }^{40} \mathbf{A r}_{\text {radiogenic }}\left(\right.$ mole g $\left.^{-1}\right)$ & ${ }^{40} \mathrm{Ar}$ radiogenic $(\%)$ & Age $( \pm 1 \sigma, M a)$ \\
\hline IM18 & QT & 91.15 & 0.85 & $5.415 \mathrm{E}-11$ & 78.99 & $36.4 \pm 0.8$ \\
\hline IM33 & OT & 83.65 & 0.94 & $5.800 \mathrm{E}-11$ & 93.85 & $35.2 \pm 0.7$ \\
\hline IM03 & QT & 33.00 & 1.02 & $6.287 \mathrm{E}-11$ & 84.56 & $35.2 \pm 0.7$ \\
\hline IM14 & QT & 90.80 & 0.90 & $5.476 \mathrm{E}-11$ & 91.82 & $34.8 \pm 0.7$ \\
\hline IM09B & QT & 92.70 & 0.83 & 4.934E-11 & 93.61 & $34.0 \pm 0.9$ \\
\hline IM20 & QT & 72.40 & 0.77 & $4.533 \mathrm{E}-11$ & 89.38 & $33.6 \pm 0.9$ \\
\hline IM16 & QT & 39.45 & 1.09 & $6.320 \mathrm{E}-11$ & 83.46 & $33.1 \pm 0.6$ \\
\hline IM19 & AOB & 37.95 & 2.19 & $1.207 \mathrm{E}-10$ & 92.63 & $31.5 \pm 0.6$ \\
\hline IM12 & OT & 27.75 & 0.84 & $3.380 \mathrm{E}-11$ & 65.64 & $23.1 \pm 0.5$ \\
\hline IM31B & B & 25.55 & 0.95 & $3.646 \mathrm{E}-11$ & 77.36 & $22.0 \pm 0.4$ \\
\hline IM32 & B & 29.75 & 1.64 & $5.828 \mathrm{E}-11$ & 65.44 & $20.4 \pm 0.4$ \\
\hline IM04 & OT & 20.50 & 1.26 & $2.151 \mathrm{E}-11$ & 51.68 & $9.8 \pm 0.2$ \\
\hline IM01 & B & 77.40 & 2.06 & $1.806 \mathrm{E}-11$ & 77.26 & $5.1 \pm 0.1$ \\
\hline IM30 & B & 72.25 & 2.40 & $5.452 \mathrm{E}-12$ & 3.247 & $1.3 \pm 0.4$ \\
\hline IM29 & B & 142.75 & 2.09 & $3.800 \mathrm{E}-13$ & 7.343 & $0.11 \pm 0.01$ \\
\hline
\end{tabular}

- Rock Types: B: Basanite; AOB: Alkali olivine basalt; OT: Olivine tholeiite; QT: Quartz tholeiite.

- Parameters for ${ }^{40} \mathrm{~K}: \lambda=5.543 \times 10^{-10} \mathrm{yr}^{-1} ; \lambda_{e}=0.581 \times 10^{-10} \mathrm{yr}^{-1} ; \lambda_{\beta}=4.962 \times 10^{-10} \mathrm{yr}^{-1} ;{ }^{40} \mathrm{~K} / \mathrm{K}=1.167 \times 10^{-4}$ atom\% (Steiger and Jäger 1977$)$.

- Standard LP-6 analyzed value is $126.5 \pm 1.2 \mathrm{Ma}$ (recommended value: $128.9 \pm 1.4 \mathrm{Ma}$ ). 
Ho. et al.

Table 2. Major (wt\%) and trace-element ( $\mathrm{ppm}$ ) compositions of basaltic rocks from the Jining region, Inner Mongolia.

\begin{tabular}{|c|c|c|c|c|c|c|c|}
\hline Sample No. & IM 01 & IM 02 & IM 03 & IM 04 & IM 05 & IM 06 & IM 07 \\
\hline Rock Type & B & OT & QT & OT & OT & OT & OT \\
\hline $\mathrm{SiO}_{2}$ & 46.13 & 49.05 & 52.79 & 50.05 & 50.81 & 50.41 & 50.63 \\
\hline $\mathrm{Al}_{2} \mathrm{O}_{3}$ & 14.69 & 14.50 & 14.60 & 14.63 & 15.40 & 14.78 & 14.81 \\
\hline$\Sigma \mathrm{FeO}^{\mathrm{a}}$ & 12.49 & 11.70 & 10.24 & 10.47 & 10.91 & 10.95 & 10.82 \\
\hline $\mathrm{MgO}$ & 6.88 & 7.73 & 7.29 & 7.48 & 7.45 & 7.87 & 6.95 \\
\hline $\mathrm{CaO}$ & 8.41 & 8.66 & 8.50 & 9.12 & 8.52 & 8.17 & 8.88 \\
\hline $\mathrm{Na}_{2} \mathrm{O}$ & 3.94 & 3.20 & 2.96 & 3.45 & 3.58 & 3.56 & 3.22 \\
\hline $\mathrm{K}_{2} \mathrm{O}$ & 2.66 & 1.32 & 1.09 & 1.49 & 1.59 & 1.52 & 1.06 \\
\hline $\mathrm{TiO}_{2}$ & 2.79 & 2.70 & 1.91 & 1.93 & 2.01 & 2.02 & 1.83 \\
\hline $\mathrm{P}_{2} \mathrm{O}_{5}$ & 1.01 & 0.44 & 0.33 & 0.37 & 0.39 & 0.37 & 0.35 \\
\hline $\mathrm{MnO}$ & 0.17 & 0.16 & 0.13 & 0.14 & 0.15 & 0.15 & 0.15 \\
\hline L.O.I. & 1.03 & 0.26 & 1.09 & 1.13 & 0.00 & 0.00 & 1.20 \\
\hline Total & 100.20 & 99.72 & 100.93 & 100.26 & 100.81 & 99.80 & 99.90 \\
\hline $\mathrm{MG}^{\mathrm{b}}$ & 53.67 & 58.15 & 59.96 & 60.04 & 58.95 & 60.18 & 57.46 \\
\hline $\mathrm{Li}$ & 12.1 & 7.6 & 4.3 & 7.0 & 7.1 & 7.2 & 6.7 \\
\hline $\mathrm{Sc}$ & 15.7 & 21.4 & 19.9 & 19.6 & 20.4 & 20.9 & 21.4 \\
\hline $\mathrm{V}$ & 181 & 194 & 148 & 157 & 161 & 164 & 160 \\
\hline $\mathrm{Cr}$ & 121 & 208 & 193 & 236 & 230 & 236 & 178 \\
\hline Co & 50 & 50 & 46 & 46 & 46 & 48 & 47 \\
\hline $\mathrm{Ni}$ & 142 & 144 & 169 & 175 & 168 & 179 & 120 \\
\hline $\mathrm{Cu}$ & 52 & 42 & 65 & 37 & 56 & 41 & 62 \\
\hline $\mathrm{Zn}$ & 166 & 117 & 127 & 119 & 112 & 119 & 117 \\
\hline $\mathrm{Rb}$ & 30.5 & 15.7 & 16.6 & 17.6 & 17.9 & 17.7 & 16.4 \\
\hline $\mathrm{Sr}$ & 977 & 492 & 446 & 515 & 527 & 532 & 465 \\
\hline $\mathrm{Y}$ & 27.5 & 27.4 & 21.3 & 21.3 & 22.1 & 21.7 & 22.2 \\
\hline $\mathrm{Zr}$ & 331 & 175 & 129 & 142 & 145 & 146 & 136 \\
\hline $\mathrm{Nb}$ & 62 & 30 & 19 & 22 & 22 & 22 & 21 \\
\hline Cs & 0.39 & 0.06 & 0.13 & 0.18 & 0.07 & 0.09 & 0.11 \\
\hline $\mathrm{Ba}$ & 438 & 265 & 250 & 342 & 351 & 342 & 274 \\
\hline $\mathrm{La}$ & 42.7 & 17.6 & 16.1 & 18.4 & 19.1 & 18.8 & 18.8 \\
\hline $\mathrm{Ce}$ & 84.9 & 36.4 & 33.2 & 37.4 & 38.7 & 38.2 & 38.0 \\
\hline $\operatorname{Pr}$ & 11.3 & 5.1 & 4.4 & 4.9 & 5.1 & 5.1 & 5.0 \\
\hline $\mathrm{Nd}$ & 45.9 & 22.8 & 19.2 & 21.0 & 21.7 & 21.6 & 21.7 \\
\hline $\mathrm{Sm}$ & 9.63 & 6.05 & 4.67 & 4.79 & 4.92 & 5.03 & 5.02 \\
\hline $\mathrm{Eu}$ & 2.81 & 1.83 & 1.52 & 1.50 & 1.55 & 1.55 & 1.57 \\
\hline $\mathrm{Tb}$ & 1.23 & 0.98 & 0.73 & 0.71 & 0.76 & 0.74 & 0.77 \\
\hline Dy & 5.73 & 5.14 & 3.88 & 3.84 & 3.99 & 3.98 & 4.06 \\
\hline Но & 0.93 & 0.91 & 0.73 & 0.71 & 0.73 & 0.73 & 0.74 \\
\hline $\mathrm{Er}$ & 2.17 & 2.30 & 1.76 & 1.76 & 1.86 & 1.84 & 1.90 \\
\hline $\mathrm{Tm}$ & 0.28 & 0.33 & 0.26 & 0.27 & 0.29 & 0.28 & 0.29 \\
\hline $\mathrm{Yb}$ & 1.49 & 1.78 & 1.46 & 1.57 & 1.59 & 1.57 & 1.56 \\
\hline $\mathrm{Lu}$ & 0.20 & 0.25 & 0.20 & 0.23 & 0.23 & 0.23 & 0.23 \\
\hline $\mathrm{Hf}$ & 6.86 & 4.11 & 3.16 & 3.35 & 3.44 & 3.46 & 3.28 \\
\hline $\mathrm{Ta}$ & 4.18 & 1.96 & 1.23 & 1.44 & 1.45 & 1.44 & 1.49 \\
\hline $\mathrm{Pb}$ & 4.5 & 1.5 & 5.1 & 4.9 & 3.6 & 3.5 & 2.7 \\
\hline Th & 4.80 & 2.12 & 1.75 & 1.94 & 2.01 & 1.94 & 2.04 \\
\hline $\mathrm{U}$ & 1.47 & 0.60 & 0.43 & 0.66 & 0.37 & 0.39 & 0.57 \\
\hline$(\mathrm{La} / \mathrm{Yb})_{\mathrm{N}}$ & 20.56 & 7.09 & 7.91 & 8.41 & 8.62 & 8.59 & 8.64 \\
\hline
\end{tabular}

Note:

${ }^{a} \mathrm{SFeO}=\mathrm{SFe}_{2} \mathrm{O}_{3} / 1.1$, and assuming that $\mathrm{Fe}_{2} \mathrm{O}_{3}=0.2 \mathrm{FeO}$.

${ }^{b} \mathrm{MG}(\mathrm{Mg}$-value $)=100 \mathrm{Mg} /\left(\mathrm{Mg}+\mathrm{Fe}^{+2}\right)$. 
Table 2. (Continued)

\begin{tabular}{|c|c|c|c|c|c|c|c|}
\hline Sample No. & IM 08 & IM 09A & IM 09B & IM 10 & IM 11 & IM 12 & IM 13 \\
\hline Rock Type & OT & OT & QT & OT & OT & OT & QT \\
\hline $\mathrm{SiO}_{2}$ & 51.12 & 50.95 & 53.32 & 51.21 & 51.33 & 50.69 & 51.44 \\
\hline $\mathrm{Al}_{2} \mathrm{O}_{3}$ & 14.66 & 14.77 & 15.04 & 14.78 & 15.22 & 14.67 & 14.59 \\
\hline$\Sigma \mathrm{FeO}$ & 10.69 & 10.66 & 10.20 & 10.19 & 10.77 & 10.66 & 10.51 \\
\hline $\mathrm{MgO}$ & 6.67 & 6.70 & 6.14 & 6.40 & 6.52 & 6.90 & 6.40 \\
\hline $\mathrm{CaO}$ & 9.03 & 9.02 & 8.96 & 8.78 & 8.75 & 8.83 & 8.60 \\
\hline $\mathrm{Na}_{2} \mathrm{O}$ & 3.10 & 3.11 & 3.11 & 3.26 & 3.64 & 3.18 & 3.18 \\
\hline $\mathrm{K}_{2} \mathrm{O}$ & 0.88 & 0.84 & 0.91 & 1.12 & 0.99 & 0.94 & 0.93 \\
\hline $\mathrm{TiO}_{2}$ & 1.84 & 1.89 & 1.58 & 1.87 & 1.94 & 1.89 & 1.86 \\
\hline $\mathrm{P}_{2} \mathrm{O}_{5}$ & 0.34 & 0.34 & 0.23 & 0.35 & 0.33 & 0.34 & 0.32 \\
\hline $\mathrm{MnO}$ & 0.14 & 0.14 & 0.13 & 0.13 & 0.14 & 0.14 & 0.14 \\
\hline L.O.I. & 2.19 & 2.40 & 1.20 & 1.97 & 1.20 & 1.20 & 1.18 \\
\hline Total & 100.66 & 100.82 & 100.82 & 100.06 & 100.83 & 99.44 & 99.15 \\
\hline MG & 56.75 & 56.93 & 55.87 & 56.91 & 56.01 & 57.65 & 56.15 \\
\hline $\mathrm{Li}$ & 6.1 & 6.2 & 6.2 & 6.6 & 6.1 & 5.8 & 6.1 \\
\hline $\mathrm{Sc}$ & 19.9 & 20.8 & 19.7 & 19.8 & 33.6 & 19.5 & 21.2 \\
\hline $\mathrm{V}$ & 162 & 166 & 150 & 162 & 158 & 162 & 166 \\
\hline $\mathrm{Cr}$ & 171 & 162 & 145 & 172 & 153 & 161 & 157 \\
\hline Co & 45 & 45 & 44 & 47 & 44 & 45 & 46 \\
\hline $\mathrm{Ni}$ & 110 & 112 & 119 & 120 & 115 & 115 & 118 \\
\hline $\mathrm{Cu}$ & 60 & 61 & 64 & 60 & 72 & 62 & 68 \\
\hline $\mathrm{Zn}$ & 110 & 116 & 110 & 109 & 131 & 114 & 118 \\
\hline $\mathrm{Rb}$ & 10.9 & 9.2 & 14.3 & 16.4 & 12.8 & 14.3 & 13.0 \\
\hline $\mathrm{Sr}$ & 477 & 484 & 373 & 464 & 432 & 470 & 445 \\
\hline $\mathrm{Y}$ & 21.7 & 22.5 & 19.2 & 22.0 & 22.2 & 21.9 & 22.8 \\
\hline $\mathrm{Zr}$ & 135 & 136 & 102 & 133 & 152 & 133 & 135 \\
\hline $\mathrm{Nb}$ & 21 & 22 & 14 & 21 & 20 & 21 & 20 \\
\hline Cs & 0.12 & 0.12 & 0.10 & 0.09 & 0.14 & 0.18 & 0.13 \\
\hline $\mathrm{Ba}$ & 271 & 273 & 218 & 269 & 262 & 271 & 256 \\
\hline $\mathrm{La}$ & 18.4 & 18.9 & 12.5 & 19.0 & 17.7 & 18.2 & 18.0 \\
\hline $\mathrm{Ce}$ & 37.5 & 38.5 & 25.9 & 38.7 & 36.2 & 37.4 & 36.7 \\
\hline $\operatorname{Pr}$ & 5.0 & 5.1 & 3.4 & 5.1 & 4.7 & 4.9 & 4.8 \\
\hline $\mathrm{Nd}$ & 21.4 & 21.9 & 15.2 & 21.9 & 20.6 & 21.4 & 20.9 \\
\hline $\mathrm{Sm}$ & 4.94 & 5.12 & 3.78 & 5.01 & 4.85 & 4.95 & 4.99 \\
\hline $\mathrm{Eu}$ & 1.52 & 1.56 & 1.23 & 1.55 & 1.50 & 1.54 & 1.55 \\
\hline $\mathrm{Tb}$ & 0.75 & 0.77 & 0.62 & 0.76 & 0.77 & 0.74 & 0.79 \\
\hline Dy & 3.97 & 4.16 & 3.46 & 4.06 & 4.13 & 4.02 & 4.17 \\
\hline Ho & 0.73 & 0.74 & 0.65 & 0.74 & 0.76 & 0.75 & 0.76 \\
\hline $\mathrm{Er}$ & 1.82 & 1.88 & 1.60 & 1.84 & 1.86 & 1.82 & 1.94 \\
\hline $\mathrm{Tm}$ & 0.27 & 0.28 & 0.25 & 0.27 & 0.28 & 0.26 & 0.28 \\
\hline $\mathrm{Yb}$ & 1.52 & 1.51 & 1.37 & 1.58 & 1.55 & 1.52 & 1.59 \\
\hline $\mathrm{Lu}$ & 0.22 & 0.22 & 0.21 & 0.22 & 0.22 & 0.22 & 0.22 \\
\hline $\mathrm{Hf}$ & 3.19 & 3.24 & 2.52 & 3.19 & 3.67 & 3.15 & 3.26 \\
\hline $\mathrm{Ta}$ & 1.36 & 1.39 & 1.06 & 1.32 & 1.16 & 1.29 & 1.34 \\
\hline $\mathrm{Pb}$ & 2.6 & 2.5 & 1.9 & 11.7 & 5.4 & 2.2 & 5.4 \\
\hline Th & 2.00 & 2.03 & 1.31 & 2.04 & 1.92 & 1.93 & 1.92 \\
\hline $\mathrm{U}$ & 0.56 & 0.57 & 0.32 & 0.57 & 0.53 & 0.55 & 0.53 \\
\hline$(\mathrm{La} / \mathrm{Yb})_{\mathrm{N}}$ & 8.68 & 8.98 & 6.54 & 8.63 & 8.19 & 8.59 & 8.12 \\
\hline
\end{tabular}


Ho. et al.

Table 2. (Continued)

\begin{tabular}{|c|c|c|c|c|c|c|c|}
\hline Sample No. & IM 14 & IM 15 & IM 16 & IM 17 & IM 18 & IM 19 & IM 20 \\
\hline Rock Type & QT & QT & QT & QT & QT & AOB & QT \\
\hline $\mathrm{SiO}_{2}$ & 53.04 & 52.68 & 52.78 & 52.36 & 52.66 & 49.95 & 53.54 \\
\hline $\mathrm{Al}_{2} \mathrm{O}_{3}$ & 14.85 & 14.80 & 14.56 & 14.76 & 15.51 & 14.58 & 14.86 \\
\hline$\Sigma \mathrm{FeO}$ & 9.80 & 9.66 & 9.81 & 9.34 & 9.58 & 9.31 & 9.86 \\
\hline $\mathrm{MgO}$ & 6.10 & 6.30 & 6.56 & 6.69 & 6.39 & 4.91 & 6.08 \\
\hline $\mathrm{CaO}$ & 8.52 & 8.39 & 8.25 & 8.32 & 8.57 & 8.97 & 8.47 \\
\hline $\mathrm{Na}_{2} \mathrm{O}$ & 2.93 & 3.03 & 3.10 & 3.06 & 3.18 & 3.46 & 3.05 \\
\hline $\mathrm{K}_{2} \mathrm{O}$ & 1.00 & 0.94 & 1.22 & 0.97 & 1.10 & 2.46 & 0.83 \\
\hline $\mathrm{TiO}_{2}$ & 1.57 & 1.59 & 1.74 & 1.67 & 1.67 & 2.19 & 1.61 \\
\hline $\mathrm{P}_{2} \mathrm{O}_{5}$ & 0.23 & 0.23 & 0.29 & 0.30 & 0.31 & 0.60 & 0.23 \\
\hline $\mathrm{MnO}$ & 0.14 & 0.11 & 0.13 & 0.11 & 0.13 & 0.12 & 0.13 \\
\hline L.O.I. & 0.99 & 2.08 & 1.45 & 2.03 & 0.67 & 2.67 & 1.00 \\
\hline Total & 99.17 & 99.81 & 99.89 & 99.61 & 99.77 & 99.22 & 99.66 \\
\hline MG & 56.69 & 57.83 & 58.44 & 60.10 & 58.38 & 52.59 & 56.46 \\
\hline $\mathrm{Li}$ & 5.3 & 4.8 & 5.0 & 5.3 & 6.9 & 10.9 & 5.0 \\
\hline $\mathrm{Sc}$ & 19.3 & 19.3 & 18.4 & 17.3 & 17.9 & 15.5 & 20.3 \\
\hline $\mathrm{V}$ & 153 & 113 & 153 & 149 & 149 & 169 & 159 \\
\hline $\mathrm{Cr}$ & 145 & 114 & 165 & 185 & 191 & 195 & 143 \\
\hline Co & 45 & 33 & 46 & 44 & 45 & 38 & 47 \\
\hline $\mathrm{Ni}$ & 115 & 118 & 139 & 121 & 124 & 92 & 116 \\
\hline $\mathrm{Cu}$ & 63 & 69 & 63 & 53 & 47 & 54 & 64 \\
\hline $\mathrm{Zn}$ & 109 & 106 & 113 & 103 & 110 & 123 & 111 \\
\hline $\mathrm{Rb}$ & 15.3 & 13.1 & 16.6 & 12.5 & 15.2 & 35.6 & 15.6 \\
\hline $\mathrm{Sr}$ & 393 & 283 & 473 & 480 & 474 & 779 & 402 \\
\hline $\mathrm{Y}$ & 19.7 & 14.8 & 20.1 & 18.8 & 20.1 & 19.3 & 21.2 \\
\hline $\mathrm{Zr}$ & 105 & 79 & 127 & 123 & 122 & 175 & 109 \\
\hline $\mathrm{Nb}$ & 14 & 11 & 19 & 21 & 21 & 40 & 14 \\
\hline Cs & 0.11 & 0.10 & 0.15 & 0.09 & 0.14 & 0.56 & 0.11 \\
\hline $\mathrm{Ba}$ & 323 & 175 & 300 & 271 & 287 & 506 & 221 \\
\hline $\mathrm{La}$ & 12.8 & 9.3 & 17.7 & 16.5 & 18.5 & 29.3 & 13.4 \\
\hline $\mathrm{Ce}$ & 26.4 & 19.0 & 35.5 & 33.1 & 33.9 & 57.3 & 27.8 \\
\hline $\operatorname{Pr}$ & 3.5 & 2.6 & 4.6 & 4.3 & 4.8 & 7.5 & 3.7 \\
\hline $\mathrm{Nd}$ & 15.7 & 11.6 & 19.6 & 18.7 & 20.6 & 30.2 & 16.5 \\
\hline $\mathrm{Sm}$ & 3.88 & 2.98 & 4.56 & 4.35 & 4.73 & 6.38 & 4.04 \\
\hline $\mathrm{Eu}$ & 1.26 & 0.98 & 1.43 & 1.4 & 1.47 & 1.88 & 1.31 \\
\hline $\mathrm{Tb}$ & 0.64 & 0.48 & 0.69 & 0.65 & 0.71 & 0.81 & 0.69 \\
\hline Dy & 3.53 & 2.75 & 3.73 & 3.46 & 3.74 & 3.86 & 3.77 \\
\hline Но & 0.66 & 0.48 & 0.67 & 0.63 & 0.68 & 0.65 & 0.70 \\
\hline $\mathrm{Er}$ & 1.67 & 1.29 & 1.64 & 1.59 & 1.68 & 1.55 & 1.77 \\
\hline $\mathrm{Tm}$ & 0.25 & 0.19 & 0.25 & 0.23 & 0.25 & 0.22 & 0.27 \\
\hline $\mathrm{Yb}$ & 1.40 & 1.10 & 1.36 & 1.29 & 1.36 & 1.17 & 1.49 \\
\hline $\mathrm{Lu}$ & 0.21 & 0.15 & 0.20 & 0.19 & 0.19 & 0.17 & 0.21 \\
\hline $\mathrm{Hf}$ & 2.62 & 1.90 & 3.06 & 2.92 & 2.92 & 3.97 & 2.65 \\
\hline $\mathrm{Ta}$ & 0.89 & 0.63 & 1.20 & 1.29 & 1.31 & 2.56 & 0.85 \\
\hline $\mathrm{Pb}$ & 2.1 & 1.6 & 2.7 & 2.3 & 3.6 & 9.7 & 1.7 \\
\hline Th & 1.36 & 0.99 & 1.98 & 2.56 & 2.73 & 4.14 & 1.39 \\
\hline $\mathrm{U}$ & 0.33 & 0.24 & 0.59 & 0.63 & 0.59 & 1.20 & 0.34 \\
\hline$(\mathrm{La} / \mathrm{Yb})_{\mathrm{N}}$ & 6.56 & 6.06 & 9.34 & 9.17 & 9.76 & 17.96 & 6.45 \\
\hline
\end{tabular}


Table 2. (Continued)

\begin{tabular}{|c|c|c|c|c|c|c|c|}
\hline Sample No. & IM 21 & IM 22 & IM 23 & IM 24A & IM 25 & IM 26 & IM 27 \\
\hline Rock Type & QT & QT & QT & QT & QT & QT & OT \\
\hline $\mathrm{SiO}_{2}$ & 52.48 & 53.83 & 52.37 & 52.84 & 52.06 & 51.81 & 52.19 \\
\hline $\mathrm{Al}_{2} \mathrm{O}_{3}$ & 14.66 & 14.67 & 14.76 & 15.09 & 14.51 & 14.57 & 15.16 \\
\hline$\Sigma \mathrm{FeO}$ & 10.02 & 9.72 & 10.18 & 10.02 & 9.79 & 9.33 & 9.62 \\
\hline $\mathrm{MgO}$ & 6.15 & 5.48 & 5.77 & 6.01 & 6.82 & 6.75 & 6.66 \\
\hline $\mathrm{CaO}$ & 8.76 & 8.69 & 8.90 & 8.95 & 8.10 & 8.59 & 8.58 \\
\hline $\mathrm{Na}_{2} \mathrm{O}$ & 3.03 & 2.99 & 3.01 & 3.05 & 3.19 & 3.07 & 3.25 \\
\hline $\mathrm{K}_{2} \mathrm{O}$ & 0.79 & 0.89 & 0.86 & 0.92 & 1.17 & 0.98 & 1.27 \\
\hline $\mathrm{TiO}_{2}$ & 1.59 & 1.58 & 1.56 & 1.61 & 1.79 & 1.74 & 1.75 \\
\hline $\mathrm{P}_{2} \mathrm{O}_{5}$ & 0.24 & 0.22 & 0.24 & 0.24 & 0.29 & 0.31 & 0.32 \\
\hline $\mathrm{MnO}$ & 0.14 & 0.12 & 0.14 & 0.14 & 0.13 & 0.12 & 0.13 \\
\hline L.O.I. & 1.49 & 1.33 & 1.36 & 0.99 & 1.51 & 2.30 & 0.68 \\
\hline Total & 99.35 & 99.52 & 99.15 & 99.86 & 99.36 & 99.57 & 99.61 \\
\hline MG & 56.35 & 54.25 & 54.38 & 55.78 & 59.43 & 60.34 & 59.28 \\
\hline $\mathrm{Li}$ & 4.9 & 5.3 & 5.4 & 5.3 & 5.7 & 5.6 & 7.2 \\
\hline $\mathrm{Sc}$ & 18.5 & 18.5 & 19.7 & 20.4 & 18.0 & 17.3 & 18.1 \\
\hline $\mathrm{V}$ & 148 & 153 & 160 & 162 & 155 & 153 & 154 \\
\hline $\mathrm{Cr}$ & 132 & 159 & 153 & 216 & 197 & 210 & 196 \\
\hline Co & 43 & 46 & 46 & 47 & 47 & 43 & 45 \\
\hline $\mathrm{Ni}$ & 107 & 130 & 121 & 120 & 151 & 123 & 124 \\
\hline $\mathrm{Cu}$ & 61 & 69 & 65 & 65 & 65 & 52 & 55 \\
\hline $\mathrm{Zn}$ & 104 & 110 & 104 & 116 & 109 & 99 & 104 \\
\hline $\mathrm{Rb}$ & 13.4 & 15.2 & 15.2 & 16.3 & 16.8 & 10.7 & 22.7 \\
\hline $\mathrm{Sr}$ & 400 & 393 & 417 & 411 & 493 & 474 & 489 \\
\hline $\mathrm{Y}$ & 19.6 & 19.6 & 21.2 & 21.4 & 20.0 & 18.8 & 19.2 \\
\hline $\mathrm{Zr}$ & 99 & 102 & 110 & 109 & 124 & 123 & 129 \\
\hline $\mathrm{Nb}$ & 13 & 13 & 14 & 14 & 19 & 21 & 22 \\
\hline Cs & 0.09 & 0.12 & 0.11 & 0.12 & 0.14 & 0.07 & 0.23 \\
\hline $\mathrm{Ba}$ & 217 & 226 & 236 & 234 & 307 & 280 & 284 \\
\hline $\mathrm{La}$ & 12.4 & 12.5 & 13.5 & 14.0 & 17.8 & 16.8 & 17.6 \\
\hline $\mathrm{Ce}$ & 25.9 & 25.9 & 28.2 & 29.0 & 35.3 & 33.9 & 35.5 \\
\hline $\operatorname{Pr}$ & 3.4 & 3.4 & 3.7 & 3.8 & 4.6 & 4.4 & 4.6 \\
\hline $\mathrm{Nd}$ & 15.3 & 15.3 & 16.6 & 16.8 & 20.1 & 18.8 & 19.6 \\
\hline $\mathrm{Sm}$ & 3.80 & 3.76 & 4.07 & 4.22 & 4.61 & 4.43 & 4.58 \\
\hline $\mathrm{Eu}$ & 1.24 & 1.26 & 1.33 & 1.35 & 1.48 & 1.41 & 1.44 \\
\hline $\mathrm{Tb}$ & 0.63 & 0.64 & 0.69 & 0.69 & 0.71 & 0.66 & 0.69 \\
\hline Dy & 3.53 & 3.56 & 3.82 & 3.83 & 3.80 & 3.50 & 3.62 \\
\hline Но & 0.67 & 0.66 & 0.70 & 0.72 & 0.66 & 0.64 & 0.65 \\
\hline Er & 1.67 & 1.66 & 1.76 & 1.82 & 1.69 & 1.56 & 1.58 \\
\hline $\mathrm{Tm}$ & 0.25 & 0.25 & 0.28 & 0.27 & 0.25 & 0.23 & 0.23 \\
\hline $\mathrm{Yb}$ & 1.42 & 1.42 & 1.50 & 1.49 & 1.43 & 1.31 & 1.33 \\
\hline $\mathrm{Lu}$ & 0.20 & 0.20 & 0.22 & 0.22 & 0.20 & 0.18 & 0.19 \\
\hline $\mathrm{Hf}$ & 2.42 & 2.48 & 2.68 & 2.72 & 3.02 & 2.96 & 3.13 \\
\hline $\mathrm{Ta}$ & 0.79 & 0.83 & 0.83 & 0.90 & 1.16 & 1.29 & 1.37 \\
\hline $\mathrm{Pb}$ & 2.7 & 4.1 & 1.7 & 1.6 & 3.0 & 2.4 & 2.9 \\
\hline Th & 1.29 & 1.29 & 1.40 & 1.46 & 1.96 & 2.53 & 2.88 \\
\hline $\mathrm{U}$ & 0.32 & 0.33 & 0.34 & 0.38 & 0.66 & 0.64 & 0.72 \\
\hline$(\mathrm{La} / \mathrm{Yb})_{\mathrm{N}}$ & 6.26 & 6.31 & 6.46 & 6.74 & 8.93 & 9.20 & 9.49 \\
\hline
\end{tabular}


Table 2. (Continued)

\begin{tabular}{|c|c|c|c|c|c|c|c|c|}
\hline Sample No. & IM 28 & IM 29 & IM 30 & IM 31A & IM 31B & IM 32 & IM 33 & IM 34 \\
\hline Rock Type & OT & B & B & OT & B & B & OT & QT \\
\hline $\mathrm{SiO}_{2}$ & 51.53 & 48.69 & 47.15 & 51.59 & 44.06 & 44.24 & 51.05 & 52.95 \\
\hline $\mathrm{Al}_{2} \mathrm{O}_{3}$ & 15.42 & 17.23 & 17.43 & 14.99 & 15.61 & 14.73 & 15.06 & 14.59 \\
\hline$\Sigma \mathrm{FeO}$ & 11.02 & 11.14 & 10.66 & 10.57 & 11.13 & 11.46 & 10.61 & 10.35 \\
\hline $\mathrm{MgO}$ & 6.23 & 4.29 & 4.22 & 6.85 & 9.59 & 8.94 & 6.54 & 6.53 \\
\hline $\mathrm{CaO}$ & 9.24 & 8.12 & 8.14 & 8.57 & 9.50 & 8.69 & 8.85 & 8.47 \\
\hline $\mathrm{Na}_{2} \mathrm{O}$ & 3.18 & 4.96 & 5.56 & 3.02 & 4.02 & 2.99 & 3.09 & 3.07 \\
\hline $\mathrm{K}_{2} \mathrm{O}$ & 0.92 & 2.74 & 3.22 & 1.10 & 1.18 & 1.94 & 1.08 & 0.98 \\
\hline $\mathrm{TiO}_{2}$ & 1.71 & 2.87 & 2.83 & 1.70 & 2.62 & 2.74 & 1.81 & 1.71 \\
\hline $\mathrm{P}_{2} \mathrm{O}_{5}$ & 0.26 & 0.73 & 1.01 & 0.26 & 0.82 & 0.70 & 0.27 & 0.25 \\
\hline $\mathrm{MnO}$ & 0.15 & 0.16 & 0.15 & 0.14 & 0.18 & 0.16 & 0.13 & 0.14 \\
\hline L.O.I. & 1.29 & 0.00 & 0.02 & 0.76 & 2.18 & 3.16 & 1.76 & 0.48 \\
\hline Total & 100.95 & 100.93 & 100.39 & 99.55 & 100.89 & 99.75 & 100.25 & 99.52 \\
\hline MG & 54.32 & 44.75 & 45.43 & 57.68 & 64.44 & 62.13 & 56.45 & 57.02 \\
\hline $\mathrm{Li}$ & 5.3 & 8.8 & 9.6 & 4.7 & 7.5 & 7.5 & 4.3 & 4.9 \\
\hline $\mathrm{Sc}$ & 20.2 & 13.3 & 12.3 & 18.1 & 22.2 & 18.0 & 18.8 & 19.4 \\
\hline V & 160 & 190 & 179 & 149 & 189 & 190 & 156 & 160 \\
\hline $\mathrm{Cr}$ & 148 & 28 & 22 & 164 & 186 & 181 & 165 & 176 \\
\hline Co & 46 & 36 & 35 & 46 & 50 & 57 & 46 & 48 \\
\hline $\mathrm{Ni}$ & 115 & 25 & 24 & 139 & 157 & 174 & 134 & 143 \\
\hline $\mathrm{Cu}$ & 69 & 33 & 51 & 59 & 52 & 50 & 63 & 66 \\
\hline $\mathrm{Zn}$ & 125 & 126 & 130 & 109 & 95 & 118 & 111 & 117 \\
\hline $\mathrm{Rb}$ & 15.5 & 34.2 & 39.8 & 14.7 & 14.9 & 20.1 & 15.4 & 15.2 \\
\hline $\mathrm{Sr}$ & 414 & 888 & 1049 & 393 & 1036 & 835 & 421 & 419 \\
\hline $\mathrm{Y}$ & 21.4 & 23.1 & 25.4 & 20.6 & 28.3 & 23.3 & 21.4 & 22.5 \\
\hline $\mathrm{Zr}$ & 110 & 240 & 261 & 113 & 276 & 287 & 120 & 120 \\
\hline $\mathrm{Nb}$ & 14 & 54 & 67 & 16 & 48 & 46 & 17 & 16 \\
\hline Cs & 0.12 & 0.34 & 0.43 & 0.09 & 0.40 & 0.25 & 0.09 & 0.09 \\
\hline $\mathrm{Ba}$ & 238 & 555 & 580 & 217 & 556 & 416 & 225 & 242 \\
\hline $\mathrm{La}$ & 13.9 & 33.2 & 43.4 & 13.7 & 38.4 & 33.9 & 14.9 & 14.5 \\
\hline $\mathrm{Ce}$ & 28.9 & 63.2 & 81.2 & 28.5 & 76.7 & 68.7 & 30.7 & 29.8 \\
\hline $\operatorname{Pr}$ & 3.8 & 8.1 & 10.3 & 3.7 & 10.1 & 9.2 & 4.0 & 3.9 \\
\hline $\mathrm{Nd}$ & 16.7 & 32.4 & 40.0 & 16.4 & 40.3 & 37.1 & 17.7 & 17.5 \\
\hline $\mathrm{Sm}$ & 4.22 & 6.71 & 8.14 & 4.21 & 8.08 & 7.76 & 4.39 & 4.51 \\
\hline $\mathrm{Eu}$ & 1.34 & 2.15 & 2.47 & 1.33 & 2.39 & 2.34 & 1.43 & 1.42 \\
\hline $\mathrm{Tb}$ & 0.69 & 0.91 & 1.08 & 0.69 & 1.06 & 0.99 & 0.72 & 0.75 \\
\hline Dy & 3.82 & 4.51 & 5.07 & 3.70 & 5.21 & 4.67 & 3.92 & 4.09 \\
\hline Ho & 0.73 & 0.79 & 0.84 & 0.70 & 0.93 & 0.79 & 0.71 & 0.76 \\
\hline $\mathrm{Er}$ & 1.79 & 1.90 & 2.04 & 1.76 & 2.38 & 1.88 & 1.81 & 1.95 \\
\hline $\mathrm{Tm}$ & 0.27 & 0.27 & 0.28 & 0.26 & 0.36 & 0.27 & 0.27 & 0.29 \\
\hline $\mathrm{Yb}$ & 1.55 & 1.46 & 1.49 & 1.50 & 1.92 & 1.42 & 1.51 & 1.59 \\
\hline $\mathrm{Lu}$ & 0.22 & 0.21 & 0.20 & 0.22 & 0.28 & 0.20 & 0.22 & 0.23 \\
\hline $\mathrm{Hf}$ & 2.76 & 5.07 & 5.51 & 2.78 & 5.61 & 5.79 & 2.95 & 2.93 \\
\hline $\mathrm{Ta}$ & 0.94 & 3.31 & 4.12 & 1.05 & 3.28 & 3.22 & 1.11 & 1.04 \\
\hline $\mathrm{Pb}$ & 4.0 & 3.6 & 8.8 & 2.4 & 2.4 & 2.6 & 7.1 & 3.9 \\
\hline Th & 1.43 & 4.20 & 5.52 & 1.61 & 3.52 & 3.19 & 1.73 & 1.70 \\
\hline $\mathrm{U}$ & 0.35 & 1.21 & 1.67 & 0.36 & 1.17 & 1.18 & 0.41 & 0.37 \\
\hline$(\mathrm{La} / \mathrm{Yb})_{\mathrm{N}}$ & 6.43 & 16.31 & 20.89 & 6.55 & 14.35 & 17.12 & 7.08 & 6.54 \\
\hline
\end{tabular}


rocks are tholeiitic to alkalic and comprise four main types: quartz tholeiites, olivine tholeiites, alkali olivine basalts, and basanites. The relatively abundant rock types are quartz tholeiites and olivine tholeiites which constitute about $44 \%$ and $39 \%$, respectively, of the rock samples analyzed. Only a few basanites (14\%) and rare alkali olivine basalts (3\%) were found in the JVF, and they fall in the ternary Ne-DiOl diagram (Fig. 2a). From these major elemental data, we found that Cenozoic magmatism in the JVF was similar to that of the Leiqiong area (Ho et al. 2000), South China, which comprises voluminous tholeiites and lesser amounts of alkali basalts.

Basaltic rocks from the JVF display wide ranges of $\mathrm{SiO}_{2}(44 \sim 54 \mathrm{wt} \%), \mathrm{MgO}(4.2 \sim 9.6 \mathrm{wt} \%)$, and $\mathrm{Mg}$ val- ues (44.8 64.4), which reflect the broad variations in their chemical compositions. Figure 3 shows abundant variations of the major element oxides with $\mathrm{SiO}_{2}$ contents of basaltic rocks from the JVF. In general, the alkali basaltic samples had lower $\mathrm{SiO}_{2}$ and higher $\mathrm{TiO}_{2}$ contents than those of tholeiites; the other major elements had no obvious differences but showed a wider range of variability.

Figure 3 also shows the ranges of major elements of Hannuoba basalts for comparison. Although Jining basalts were characterized by high- $\mathrm{Ti}\left(\mathrm{Ti} / \mathrm{Y}>462, \mathrm{TiO}_{2}=1.6 \sim\right.$ 2.9 wt \%) and high $\mathrm{Na}_{2} \mathrm{O} / \mathrm{K}_{2} \mathrm{O}$ ratios (1.4 3.8), and have a within-plate nature similar to those of the Hannuoba area, as a group, the basaltic rocks from these two volcanic fields have different trends and ranges of variability in $\mathrm{SiO}_{2}, \mathrm{Al}_{2} \mathrm{O}_{3}$,

(a)

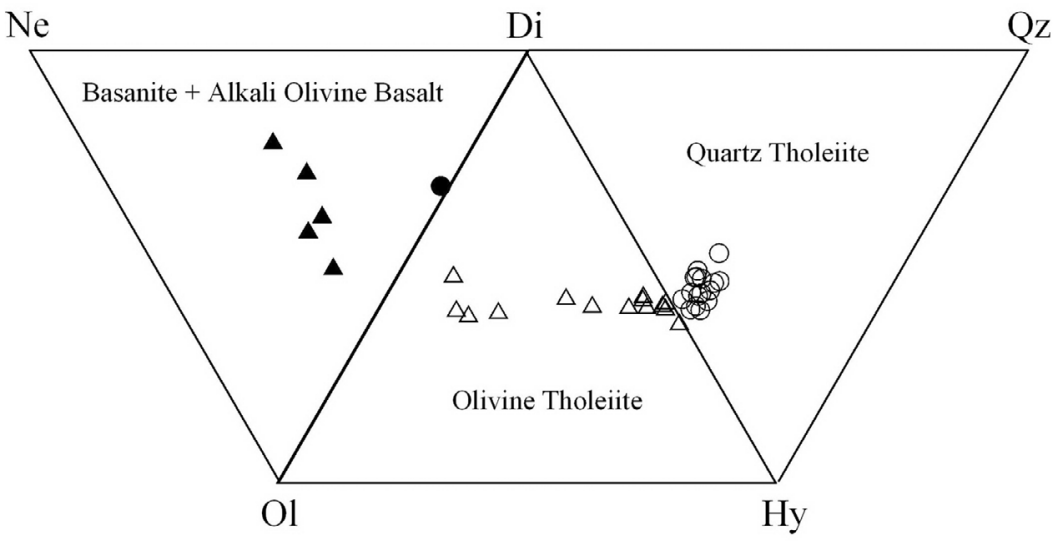

(b)

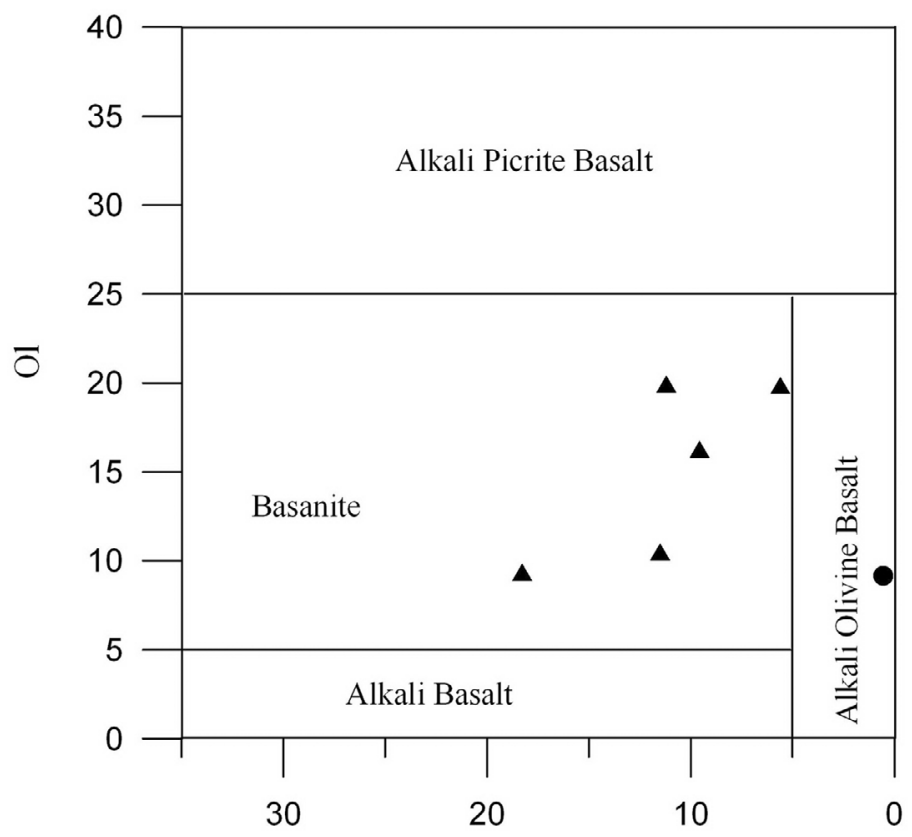

$\mathrm{Ne}$

Fig. 2. (a) Normative compositions of basaltic rocks from the Jining volcanic field (JVF). The nomenclature of volcanic rocks follows Yoder and Tilley (1962). (b) Plots of normative olivine against normative nepheline compositions of Jining alkali basalts. Field boundaries are based on those of Chih (1988). 

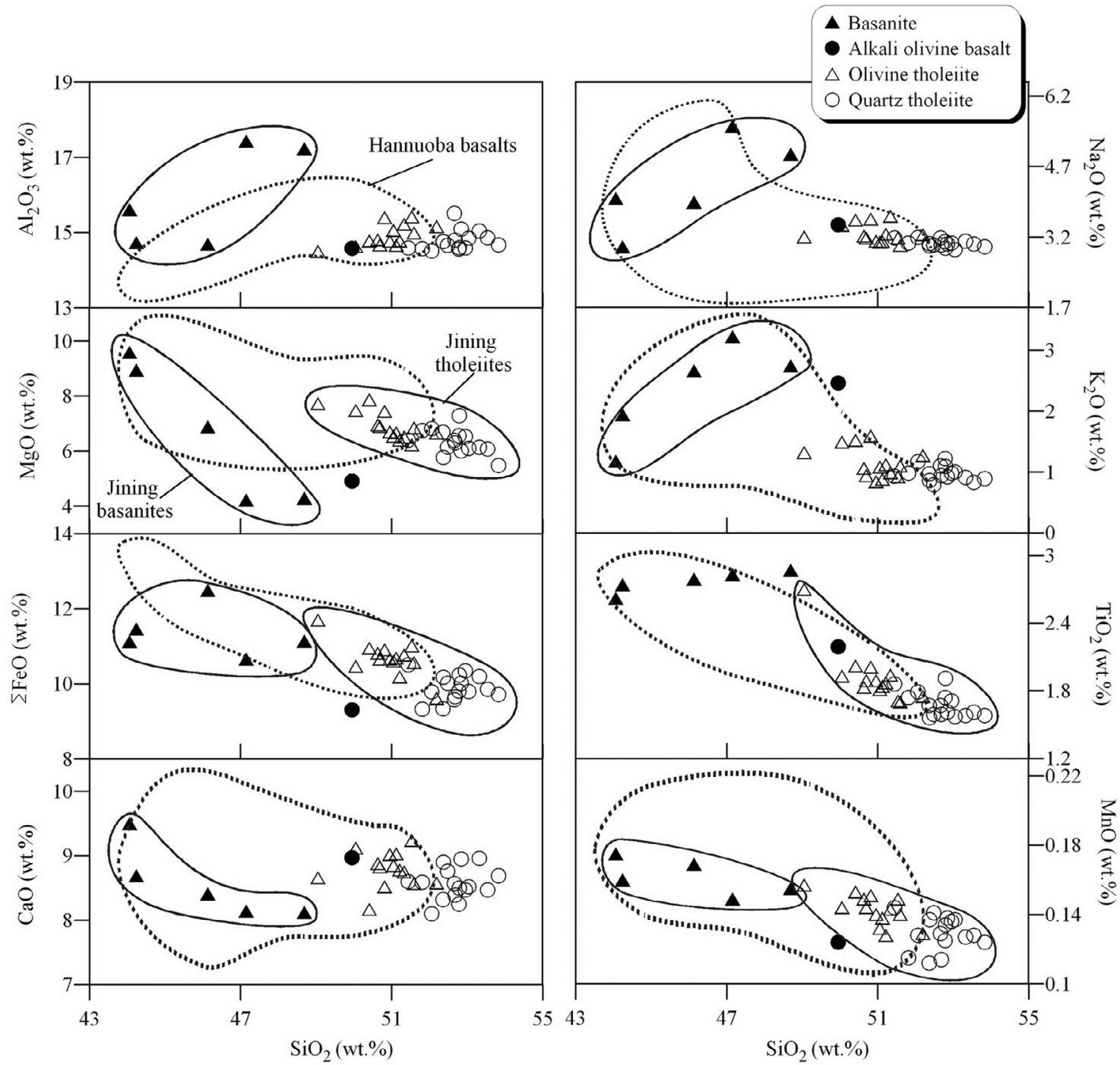

Fig. 3. Variation diagram showing major element oxides plotted against $\mathrm{SiO}_{2}$ for basaltic rocks from the Jining volcanic field (JVF). The field marked by a dashed circle depicts the distribution range of basaltic rocks from the Hannuoba volcanic field (Zhi et al. 1990).

$\mathrm{MgO}$, and $\Sigma \mathrm{FeO}$ contents (Fig. 3). It is apparent that most of the Hannuoba volcanic field includes alkali basalts and basanites, and only some lavas belong to tholeiitic basalts (Zhi et al. 1990).

\subsection{Trace Elements}

REE and other trace-element abundances of Jining basalts are also listed in Table 2. All samples exhibited subparallel smooth curves from $\mathrm{La}$ to $\mathrm{Lu}$ and displayed moderate to steep slopes with light (L)REE enrichment on chondritenormalized REE patterns (Fig. 4). Although the heavy (H) REE patterns in these samples were remarkably similar, the alkali basalts always had higher LREE/HREE ratios than the tholeiites. Therefore, the $(\mathrm{La} / \mathrm{Yb})_{\mathrm{N}}$ ratios increased with the undersaturated character from $6.1 \sim 9.8$ in tholeiites to 14.4 $\sim 20.9$ in alkali basalts (Table 2). Overall, the LREE abun- dances in alkali basalts are higher than those of E-type and $\mathrm{N}$-type mid-ocean ridge basalts (MORBs), but are comparable with ocean island basalts (OIBs, Fig. 4). The tholeiites and alkali basalts do not significant Eu anomalies indicating that plagioclase was not a major fractionating phase during the generation and evolution of these magmas.

Other incompatible element abundances in these samples also vary systematically with rock types. In general, the contents of highly incompatible elements (e.g., $\mathrm{Ba}, \mathrm{Th}, \mathrm{Nb}$, and $\mathrm{Sr}$ ) are much higher in the alkali basalts than in the tholeiites (Fig. 5). These differences are common in intraplate basaltic rocks (e.g., Ho et al. 2000, 2003; Xu et al. 2005) and most likely result from different degrees of partial melting at different depths in a heterogeneous mantle.

The analyzed samples display considerable heterogeneity in the abundances of compatible elements. For instance, $\mathrm{Cr}$ and $\mathrm{Ni}$ abundances in these basalts are moderately vari- 
able: $\mathrm{Cr}$ ranged $22 \sim 236 \mathrm{ppm}$ and Ni $24 \sim 179$ ppm. In general, the larger range of compatible trace elements are well correlated with the $\mathrm{SiO}_{2}$ contents in basanites from the JVF which is consistent with a heritage via extended fractional crystallization; in contrast, tholeiites generally display smaller variations in compatible element compositions (Fig. 5).

\subsection{Sr-Nd-Pb Isotopic Compositions}

$\mathrm{Sr}-\mathrm{Nd}-\mathrm{Pb}$ isotopic compositions of representative basaltic rocks from the JVF are given in Table 3. They ranged widely in ${ }^{87} \mathrm{Sr} /{ }^{86} \mathrm{Sr}$ and ${ }^{143} \mathrm{Nd} /{ }^{144} \mathrm{Nd}$ ratios, and reflect the iso- topic heterogeneity of basaltic rocks from the JVF. On the ${ }^{143} \mathrm{Nd} /{ }^{144} \mathrm{Nd}$ vs. ${ }^{87} \mathrm{Sr} /{ }^{86} \mathrm{Sr}$ diagram (Fig. 6), these samples have inverse corrections and fall within the $\mathrm{Sr}-\mathrm{Nd}$ "mantle array." It is apparent from Fig. 6 that the Jining basaltic rocks have distinct $\mathrm{Sr}-\mathrm{Nd}$ isotope ratios and form two groups: (1) ${ }^{87} \mathrm{Sr} /{ }^{86} \mathrm{Sr}$ ratios of $0.7035 \sim 0.7044$ and ${ }^{143} \mathrm{Nd} /{ }^{144} \mathrm{Nd}$ ratios of $0.5127 \sim 0.5129$ of basanite samples fall within the field defined by oceanic basalts and show depleted characteristics; and $(2){ }^{87} \mathrm{Sr} /{ }^{86} \mathrm{Sr}$ ratios of $0.7048 \sim 0.7054$ and ${ }^{143} \mathrm{Nd} /{ }^{144} \mathrm{Nd}$ ratios of $0.5125 \sim 0.5126$ of the tholeiite (including quartz tholeiite and olivine tholeiite) and alkali olivine basalt samples are higher and lower, respectively, than Bulk Silicate Earth estimates. It should be noted that there is a systematic

(a)

(b)

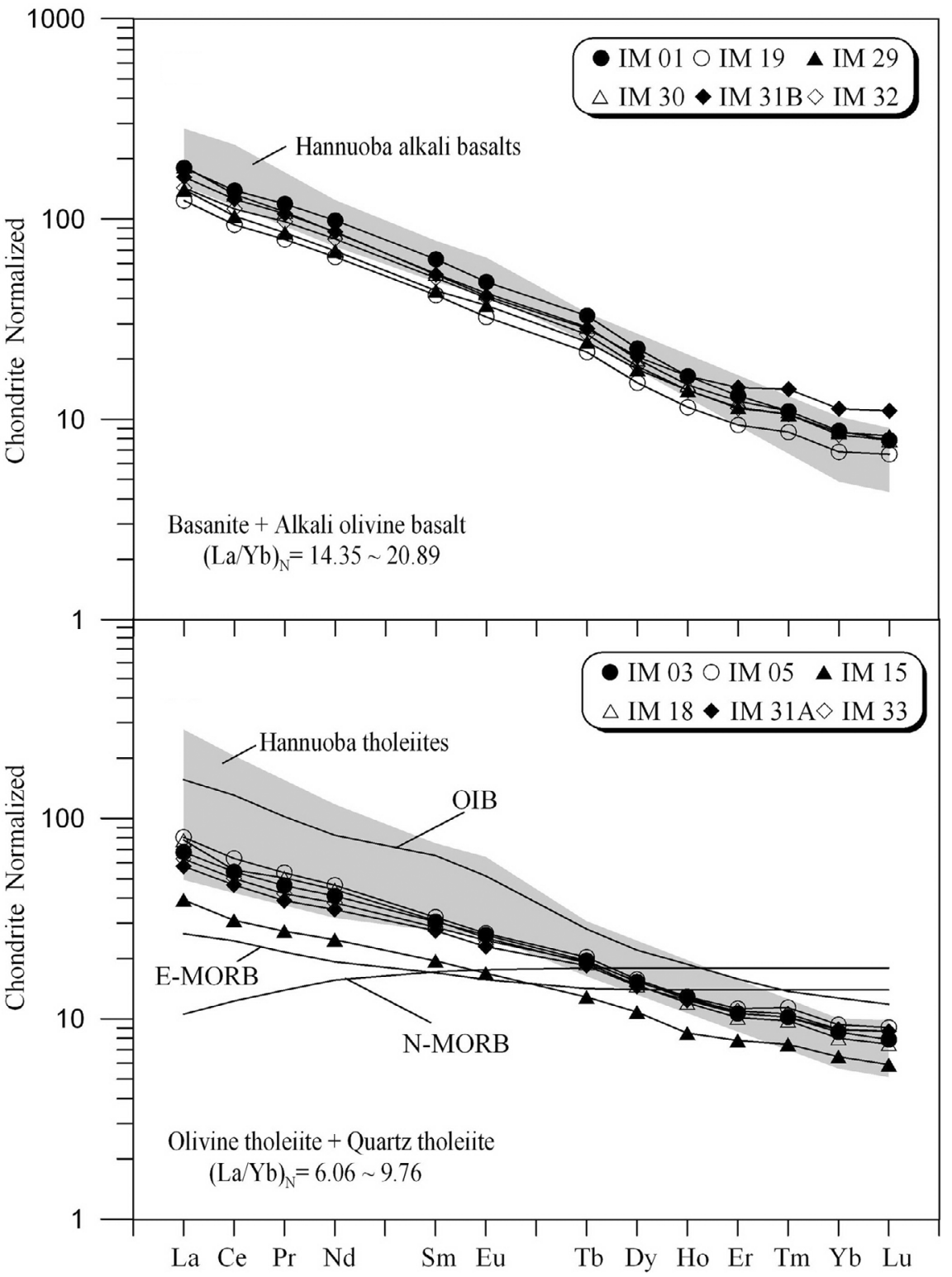

Fig. 4. (a) Chondrite-normalized diagram showing the rare-earth element (REE) patterns of representative basaltic rocks from the Jining volcanic field (JVF). (b) The average compositions of oceanic island basalt (OIB), E-type of mid-ocean ridge basalt (E-MORB), and N-MORB are plotted for comparison. Normalizing values and OIB, E-MORB, and N-MORB compositions are from Sun and McDonough (1989). 

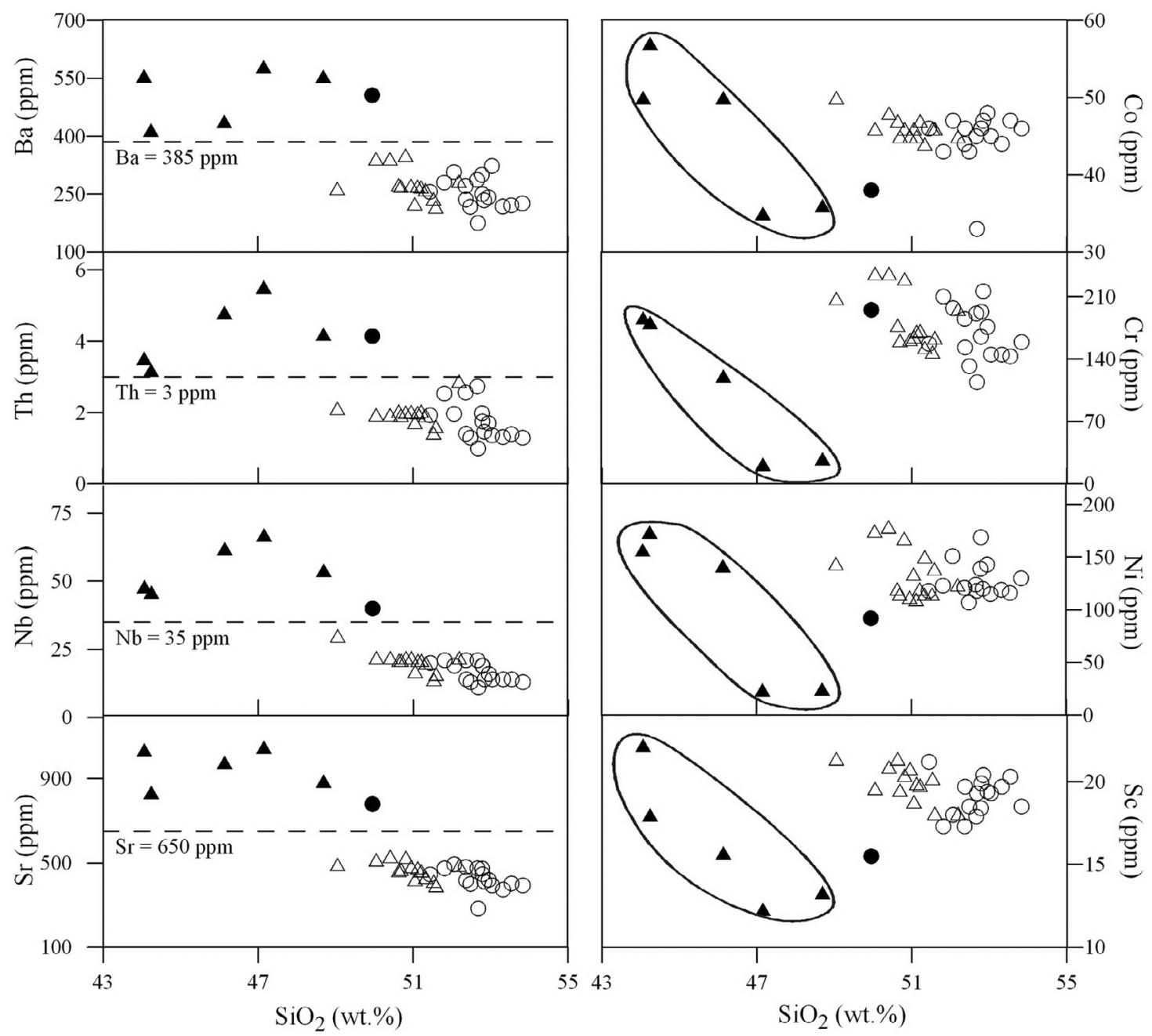

Fig. 5. Variation diagram showing incompatible (Ba, $\mathrm{Th}, \mathrm{Nb}$ and $\mathrm{Sr})$ and compatible $(\mathrm{Co}, \mathrm{Cr}, \mathrm{Ni}$ and $\mathrm{Sc})$ elements plotted against $\mathrm{SiO}{ }_{2}$ of basaltic rocks from the Jining volcanic field (JVF). The symbols are the same as those described in Fig. 3.

Table 3. Sr-Nd-Pb isotopic compositions of representative basaltic rocks from the Jining region, Inner Mongolia.

\begin{tabular}{|c|c|c|c|c|c|c|c|c|c|}
\hline Sample No. & Rock type & ${ }^{87} \mathrm{Sr} /{ }^{86} \mathrm{Sr}$ & ${ }^{143} \mathbf{N d} /{ }^{144} \mathbf{N d}$ & ${ }^{\mathrm{a}} \boldsymbol{\varepsilon} \mathbf{N d}(\mathbf{0} \mathrm{Ma})$ & ${ }^{206} \mathrm{~Pb} /{ }^{204} \mathrm{~Pb}$ & ${ }^{207} \mathrm{~Pb} /{ }^{204} \mathrm{~Pb}$ & ${ }^{208} \mathrm{~Pb} /{ }^{204} \mathrm{~Pb}$ & ${ }^{\mathrm{b}} \Delta 7 / 4$ & ${ }^{\mathrm{c}} \Delta 8 / 4$ \\
\hline IM01 & B & $0.703824 \pm 13$ & $0.512891 \pm 10$ & +4.9 & 17.902 & 15.521 & 37.887 & +8.9 & +61.6 \\
\hline IM03 & QT & $0.704785 \pm 11$ & $0.512599 \pm 12$ & -0.8 & 17.104 & 15.410 & 37.422 & +6.5 & +111.6 \\
\hline IM04 & OT & $0.705167 \pm 14$ & $0.512514 \pm 12$ & -2.4 & & & & & \\
\hline IM05 & OT & $0.705049 \pm 12$ & $0.512520 \pm 12$ & -2.3 & 17.109 & 15.411 & 37.293 & +6.5 & +98.1 \\
\hline IM09B * & QT & $0.705119 \pm 11$ & $0.512561 \pm 10$ & -1.5 & 16.909 & 15.363 & 37.084 & +3.9 & +101.4 \\
\hline $\operatorname{IM} 12 *$ & OT & $0.704874 \pm 12$ & $0.512571 \pm 10$ & -1.3 & 17.150 & 15.381 & 37.238 & +3.1 & +87.7 \\
\hline IM14* & QT & $0.705207 \pm 13$ & $0.512557 \pm 12$ & -1.6 & 16.929 & 15.359 & 37.083 & +3.3 & +98.9 \\
\hline IM15 & QT & $0.705069 \pm 11$ & $0.512576 \pm 12$ & -1.2 & & & & & \\
\hline IM16 & QT & $0.705071 \pm 12$ & $0.512554 \pm 12$ & -1.6 & 17.299 & 15.439 & 37.696 & +7.3 & +115.5 \\
\hline IM17* & QT & $0.705119 \pm 13$ & $0.512562 \pm 11$ & -1.5 & 17.179 & 15.389 & 37.737 & +3.6 & +134.1 \\
\hline IM18 & QT & $0.704926 \pm 11$ & $0.512537 \pm 10$ & -2.0 & & & & & \\
\hline IM19 & $\mathrm{AOB}$ & $0.705411 \pm 11$ & $0.512539 \pm 11$ & -1.9 & 17.438 & 15.454 & 38.068 & +7.3 & +135.8 \\
\hline
\end{tabular}


Table 3. (Continued)

\begin{tabular}{lccccccccc}
\hline Sample No. & Rock type & ${ }^{87} \mathbf{S r} /{ }^{86} \mathbf{S r}$ & ${ }^{143} \mathbf{N d} /{ }^{144} \mathbf{N d}$ & ${ }^{\mathrm{a}} \mathbf{\varepsilon N d}(\mathbf{0} \mathbf{M a})$ & ${ }^{206} \mathbf{P b} /{ }^{204} \mathbf{P b}$ & ${ }^{207} \mathbf{P b} /{ }^{204} \mathbf{P b}$ & ${ }^{208} \mathbf{P b} /{ }^{204} \mathbf{P b}$ & ${ }^{\mathrm{b}} \boldsymbol{\Delta 7 / 4}$ & ${ }^{\mathrm{c}} \mathbf{\Delta 8 / 4}$ \\
\hline IM20 & QT & $0.705179 \pm 13$ & $0.512523 \pm 12$ & -2.2 & 16.963 & 15.399 & 37.217 & +6.9 & +108.2 \\
IM29 & B & $0.704414 \pm 11$ & $0.512780 \pm 9$ & +2.8 & 17.709 & 15.524 & 38.219 & +11.3 & +118.2 \\
IM30 & B & $0.704017 \pm 13$ & $0.512840 \pm 13$ & +3.9 & 17.771 & 15.518 & 38.134 & +10.1 & +102.2 \\
IM31A * & OT & $0.705184 \pm 12$ & $0.512540 \pm 9$ & -1.9 & 16.995 & 15.353 & 37.550 & +2.0 & +137.6 \\
IM31B & B & $0.704269 \pm 12$ & $0.512703 \pm 10$ & +1.3 & 17.779 & 15.455 & 37.807 & 3.7 & +68.5 \\
IM32* & B & $0.703527 \pm 11$ & $0.512846 \pm 13$ & +4.1 & 17.973 & 15.421 & 37.826 & -1.8 & +47.0 \\
IM33 & OT & $0.705204 \pm 14$ & $0.512530 \pm 12$ & -2.1 & 17.019 & 15.381 & 37.620 & 4.5 & +141.7 \\
\hline
\end{tabular}

Note:

${ }^{a} \varepsilon N d=\left[\left({ }^{143} \mathrm{Nd} /{ }^{144} \mathrm{Nd}\right)_{\text {sample }} /\left({ }^{143} \mathrm{Nd} /{ }^{144} \mathrm{Nd}\right)_{\mathrm{CHUR}}-1\right] \times 10^{4} ;\left({ }^{143} \mathrm{Nd} /{ }^{144} \mathrm{Nd}\right)_{\mathrm{CHUR}}^{0 \mathrm{Ma}}=0.512638$.

${ }^{b} \Delta 7 / 4=\left[\left({ }^{207} \mathrm{~Pb} /{ }^{204} \mathrm{~Pb}\right)_{D S}-\left({ }^{207} \mathrm{~Pb} /{ }^{204} \mathrm{~Pb}\right)_{N H R L}\right] \times 100 ;\left({ }^{207} \mathrm{~Pb} /{ }^{204} \mathrm{~Pb}\right)_{\text {NHRL }}=0.1084\left({ }^{206} \mathrm{~Pb} /{ }^{204} \mathrm{~Pb}\right)+13.491($ Hart 1984 $)$.

${ }^{c} \Delta 8 / 4=\left[\left({ }^{208} \mathrm{~Pb} /{ }^{204} \mathrm{~Pb}\right)_{D S}-\left({ }^{208} \mathrm{~Pb} /{ }^{204} \mathrm{~Pb}\right)_{\text {NHRL }}\right] \times 100 ;\left({ }^{208} \mathrm{~Pb} /{ }^{204} \mathrm{~Pb}\right)_{\text {NHRL }}=1.209\left({ }^{206} \mathrm{~Pb} /{ }^{204} \mathrm{~Pb}\right)+15.627($ Hart 1984) .

${ }^{*} \mathrm{~Pb}$ isotope analyzed by F. K. Chen; others are analyzed by B. S. Wang.

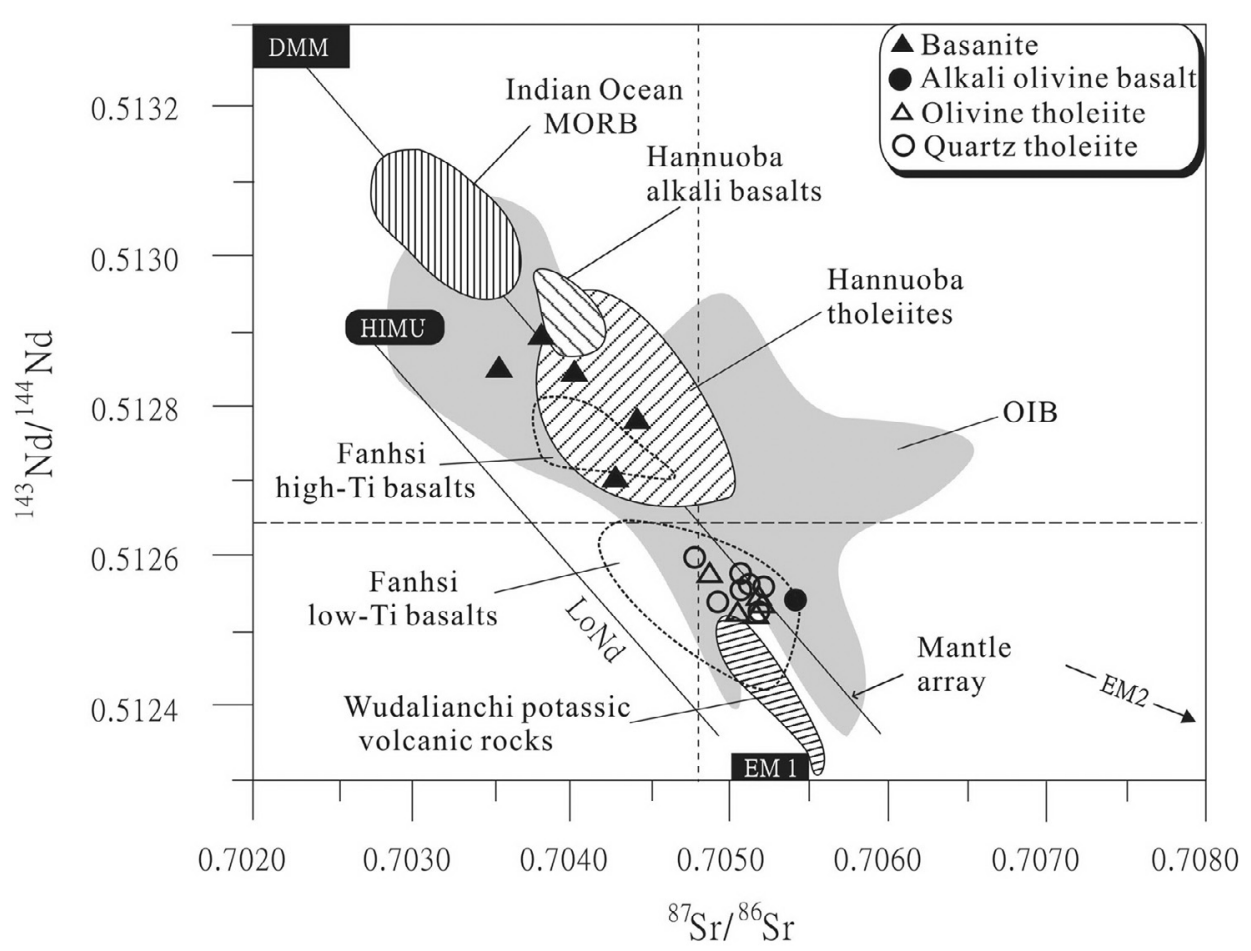

Fig. 6. Plots of ${ }^{143} \mathrm{Nd} /{ }^{144} \mathrm{Nd}$ vs. ${ }^{87} \mathrm{Sr} /{ }^{86} \mathrm{Sr}$ diagrams for the Jining basalts. Compositional fields of the Cenozoic Hannuoba (Song et al. 1990; Basu et al. 1991), Fanhsi (Tang et al. 2006), and Wudalianchi (Basu et al. 1991; Zhang et al. 1998c; Zou et al. 2003) volcanic fields are also shown in the diagrams. The Indian Ocean mid-ocean ridge basalt (MORB) and oceanic island basalt (OIB) fields are from Mahoney et al. (1989) and Zindler and Hart (1986). LoNd, Low Nd array (Hart et al. 1986).

isotopic distinction between the basanite and alkali olivine basalts. Although there are considerable fluctuations, variations in $\mathrm{Sr}-\mathrm{Nd}$ isotopic ratios of basanites roughly overlap with those of Hannuoba basalts, whereas the data points of tholeiites and alkali olivine basalts cluster with a trend toward the EM1 component of Zindler and Hart (1986), but the Nd-isotopic ratios of the Jining tholeiites are slightly higher than those of the Wudalianchi potassic volcanic rocks (Fig. 6). However, all analyzed samples are roughly collinear between the depleted MORB mantle (DMM) and enriched lithospheric mantle (EM1) type.

Lead isotopes were present in 16 basaltic rocks with the following compositional ranges: ${ }^{206} \mathrm{~Pb} /{ }^{204} \mathrm{~Pb}$ of $16.9 \sim$ $18.0,{ }^{207} \mathrm{~Pb} /{ }^{204} \mathrm{~Pb}$ of $15.4 \sim 15.5$, and ${ }^{208} \mathrm{~Pb} /{ }^{204} \mathrm{~Pb}$ of $37.1 \sim$ 
38.2 (Table 3). In the ${ }^{207} \mathrm{~Pb} /{ }^{204} \mathrm{~Pb}$ vs. ${ }^{206} \mathrm{~Pb} /{ }^{204} \mathrm{~Pb}$ diagram (Fig. 7a), the basanitic samples are displaced to significantly higher ${ }^{206} \mathrm{~Pb} / 204 \mathrm{~Pb}$ ratios compared to the tholeiites and alkali olivine basalts such that two groups of rocks lie on both sides of the $4.55-\mathrm{Ga}$ geochron. In the ${ }^{208} \mathrm{~Pb} /{ }^{204} \mathrm{~Pb}$ vs. ${ }^{206} \mathrm{~Pb} /{ }^{204} \mathrm{~Pb}$ diagram (Fig. 7b), these samples form a coherent array and lie above the North Hemisphere Reference Line (NHRL) defined by Hart (1984). The $\Delta 8 / 4 \mathrm{~Pb}$ values range $47.0 \sim 141.7$ indicating the existence of a DUPAL Pb isoto- pic anomaly (Dupré and Allègre 1983).

\section{DISCUSSION}

\subsection{Geochemical Characteristics of the Jining Basalts}

Cenozoic basaltic rocks from the western/central NCC, e.g., Hannuoba, Datong, and Taihang Mountains, were generally uncontaminated by crustal rocks (e.g., Song et al. 1990; Xu et al. 2005; Tang et al. 2006). To assess the effects
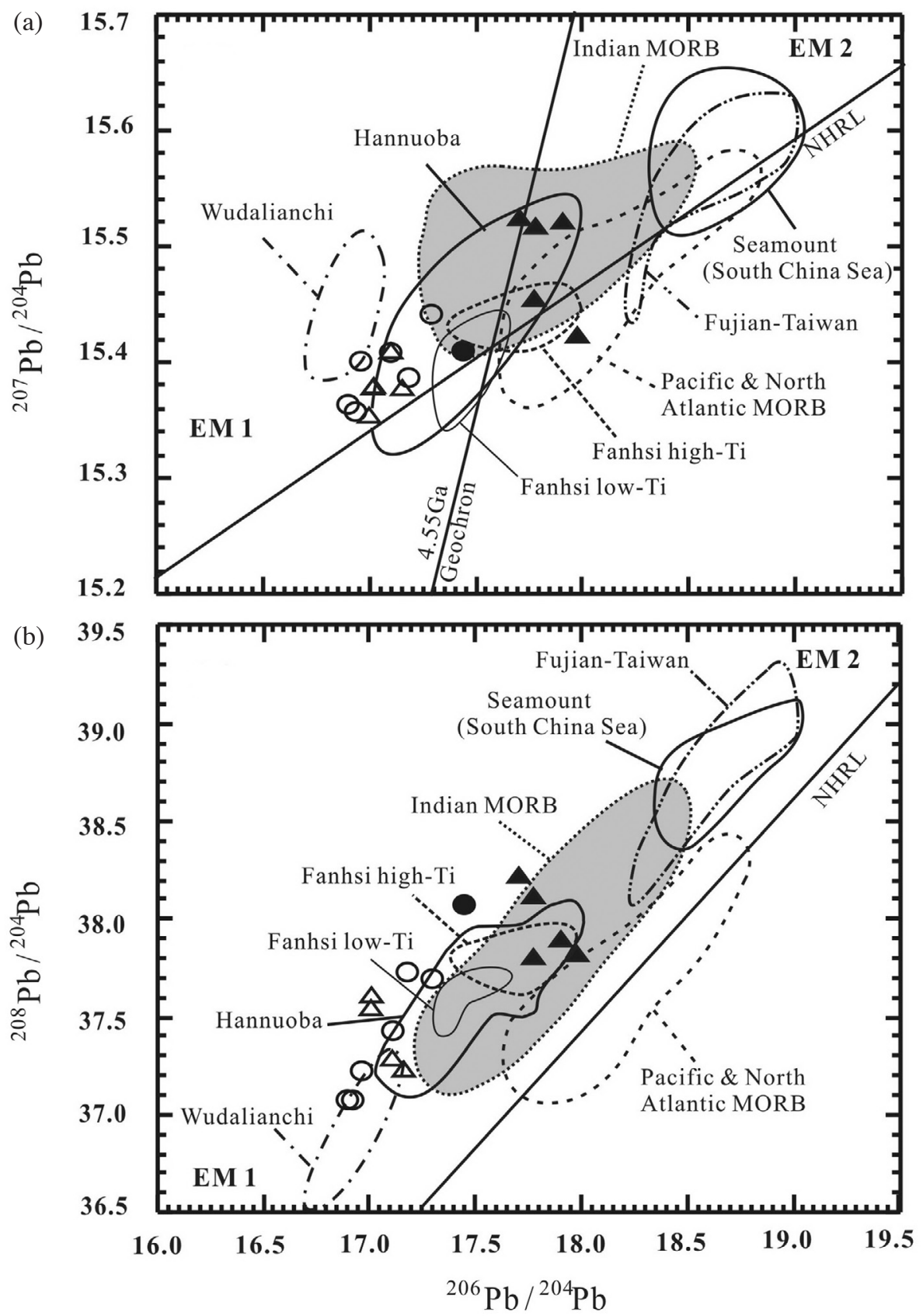

Fig. 7. ${ }^{208} \mathrm{~Pb} /{ }^{204} \mathrm{~Pb}$ and ${ }^{207} \mathrm{~Pb} /{ }^{204} \mathrm{~Pb}$ vs. ${ }^{206} \mathrm{~Pb} /{ }^{204} \mathrm{~Pb}$ diagrams for the Jining basalts. Fields for the Wudalianchi (Zhang et al. 1998c; Zou et al. 2003), Hannuoba (Song et al. 1990; Basu et al. 1991), Fanhsi (Tang et al. 2006), Fujian-Taiwan (Chung et al. 1995), South China Sea seamount (Tu et al. 1992), Indian Ocean mid-ocean ridge basalt (MORB), and Pacific and North Atlantic MORBs (Barry and Kent 1998) are shown for comparison. Fields of enriched mantle of type 1 (EM1) and EM2 are adopted from Zindler and Hart (1986). Symbols are the same as those described in Fig. 6. 
of crustal contamination during magma ascent, we used trace element and Sr-isotope ratios. In the plot of $\mathrm{Th} / \mathrm{Yb}$ vs. $\mathrm{Ta} / \mathrm{Yb}$ (Fig. 8), the Jining basalts overlap with OIBs, fall into the enriched mantle source field, and do not show high $\mathrm{Th} / \mathrm{Yb}$ ratios which might be indicative of crustal contamination. As a group, the tholeiites have higher ${ }^{87} \mathrm{Sr} /{ }^{86} \mathrm{Sr}$ ratios than the basanites, but no significant correlation between ${ }^{87} \mathrm{Sr} /{ }^{86} \mathrm{Sr}$ and $1 / \mathrm{Sr}$ can be found indicating that they were formed from mantle-derived magmas without pronounced assimilation of crustal rocks (Fig. 9). Except for the alkali olivine basalt (as discussed in Section 7.2.3), therefore, the isotopic variations of these basaltic rocks may be ascribed to mantle processes.

In the JVF, basanites have higher $\mathrm{Nb} / \mathrm{La}, \mathrm{La} / \mathrm{Ce}, \mathrm{La} /$ $\mathrm{Nd}, \mathrm{Ce} / \mathrm{Nd}$, and $\mathrm{Nd} / \mathrm{Sm}$ ratios than the tholeiites (Table 2). If these basalts had been derived from a homogeneous mantle source, the basanites may have been generated by smaller degrees of partial melting than the tholeiites; therefore, the former should have higher abundances of incompatible trace elements than the latter. However, the above-mentioned highly incompatible element ratios in the basanites and tholeiites do not vary systematically with La abundances (data not shown here), suggesting that they were not affected by partial melting or fractional crystallization processes, and may represent ratios of the mantle sources. In a mantle peridotite assemblage (olivine + orthopyroxene + clinopyroxene \pm garnet \pm spinel) the relative incompatibility of trace elements was suggested to be $\mathrm{Nb}>\mathrm{La}>\mathrm{Ce}>\mathrm{Nd}>\mathrm{Sm}$ (Sun and McDonough 1989). Thus these differences in trace-el-

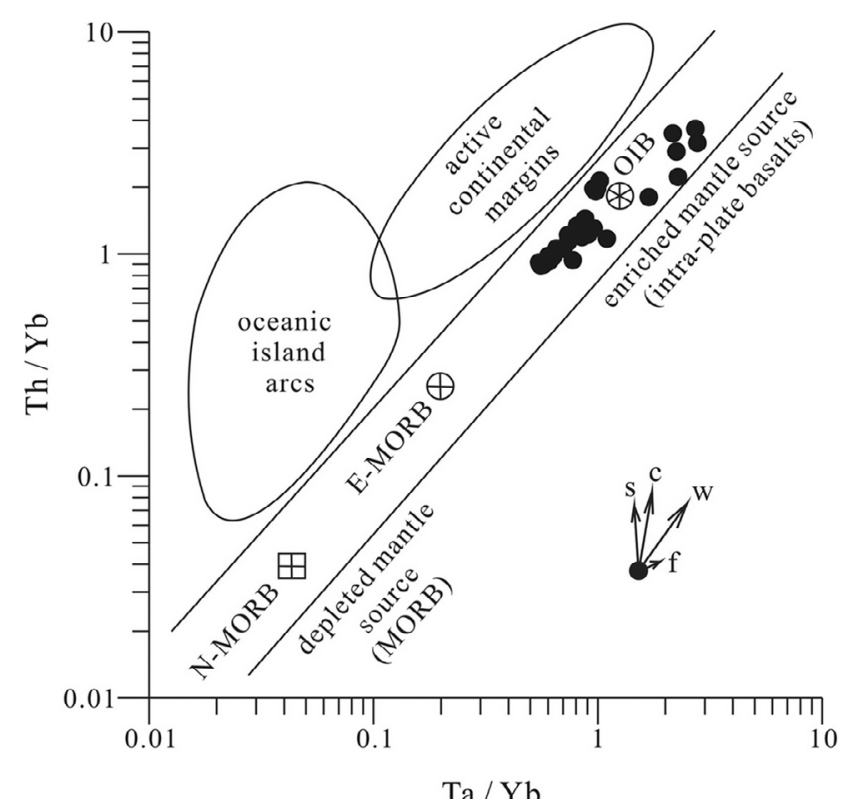

Fig. 8. Th/Yb vs. Ta/Yb plot showing the oceanic-island basalt (OIB)like character of the Cenozoic Jining basalts. Vectors for indicating the influence of subduction components (s), crustal contamination (c), within-plate enrichment (w), and fractional crystallization (f) are from Wilson (1989).

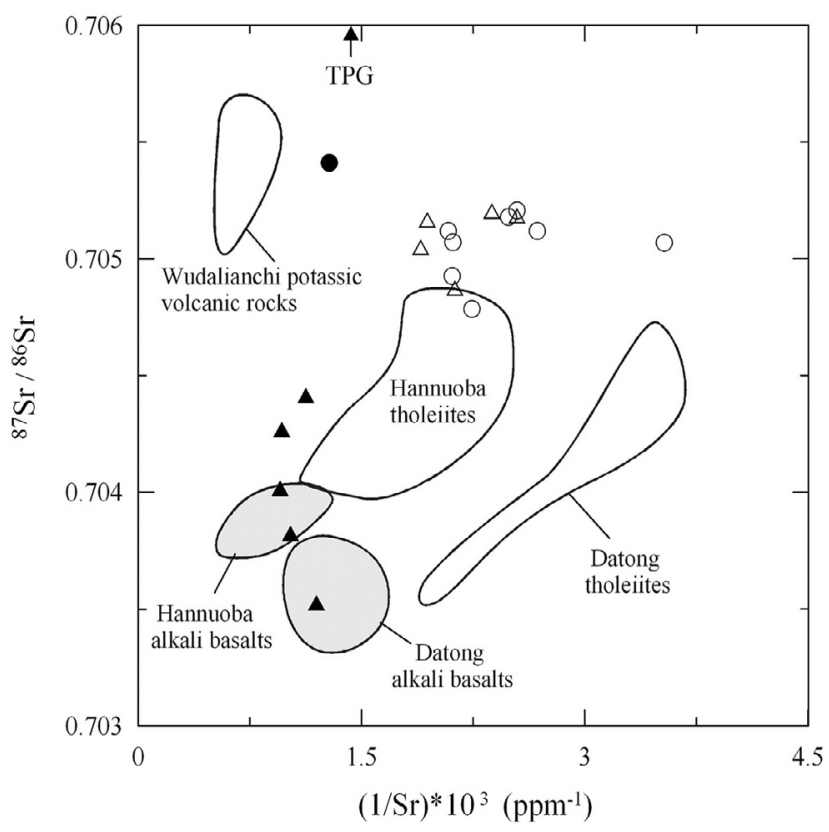

Fig. $9 .{ }^{87} \mathrm{Sr} /{ }^{86} \mathrm{Sr}$ ratios vs. reciprocal $\mathrm{Sr}$ concentrations of the Jining basalts. The data cluster of the tholeiites in the JVF partially overlaps that of Hannuoba tholeiites. Data of the Wudalianchi (Zhang et al. 1995), Hannuoba (Zhi et al. 1990), and Datong (Xu et al. 2005) volcanic fields are shown for comparison. The compositional trend of average $\mathrm{Sr}$ content $(703 \mathrm{ppm})$ and ${ }^{87} \mathrm{Sr} /{ }^{86} \mathrm{Sr}$ ratio $(0.707195)$ of twopyroxene granulite (TPG) was adopted from Zhang et al. (1998a, b). Symbols are the same as those described in Fig. 3.

ement ratios between the basanites and tholeiites may show the geochemical heterogeneity of the mantle source(s), and greater enrichment of incompatible elements in the sources of the basanites.

Figure 10 shows primitive mantle-normalized trace-element patterns for the Jining basalts. These basalts are conspicuously enriched in highly incompatible elements, which are similar to those of the Hannuoba area and OIBs. Most samples in general have slightly positive $\mathrm{K}$ anomalies, with the exception of the one basanite sample (IM31B, Fig. 10). That sample was from the earliest eruptive basanitic rock. Its negative $\mathrm{K}$ anomaly character can be interpreted as being due to K-bearing minerals in the residual phases. In addition to obvious depletions of $\mathrm{Rb}$ found in some samples, e.g., IM31B and IM32, they usually have slightly higher loss of ignition values, indicating that they may have been affected by alterations in the post-eruption stage.

\subsection{Petrogenesis of the Jining Basalts}

\subsubsection{Systematic Variations in Elemental Abundances of the Basanites}

Chemical analyses of five basanite samples show significant compositional variations (Table 2), and their $\mathrm{MgO}$ contents (4.2 9.6 wt \%) cover the entire range of the Jining samples (Fig. 3). The compatible element concentrations 


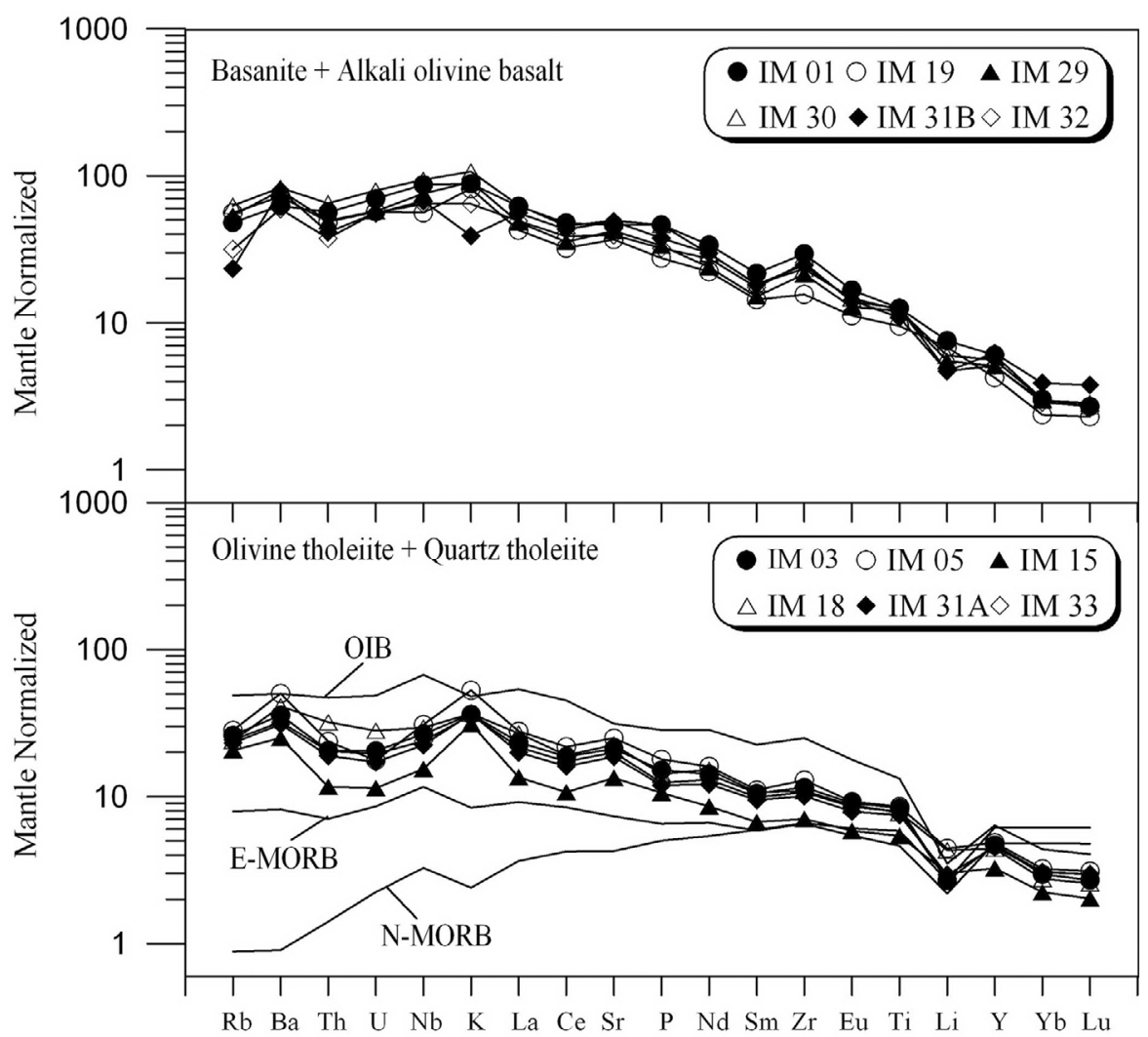

Fig. 10. Spider diagrams showing primitive mantle-normalized incompatible element abundances for selected basaltic rocks from the Jining area. N-type mid-ocean ridge basalt (N-MORB), E-MORB, and oceanic island basalt (OIB) compositions are from Sun and McDonough (1989).

display systematic correlations with $\mathrm{MgO}$ contents consistent with fractional crystallization relationships (Fig. 11). For example, the contents of Ni that have been largely concentrated in olivine are positively correlated with $\mathrm{MgO}$, whereas the contents of $\mathrm{Cr}$ that largely reside in clinopyroxene reveal a negative correlation with $\mathrm{Al}_{2} \mathrm{O}_{3} / \mathrm{CaO}$. Based on the composition of primary basalts defined by Hart and Allègre (1980), samples IM31B and IM32 containing the highest $\mathrm{MgO}, \mathrm{Ni}$, and $\mathrm{Cr}$ concentrations as well as the lowest $\mathrm{SiO}_{2}$ in the analyzed basanite samples may be closest in composition to the primary magma. Samples IM29 and IM30 on the other hand probably have undergone a larger degree of olivine and clinopyroxene fractional crystallization and represent the most evolved magma products observed.

Another important observation is that the earliest formed basanite chemically differs from the latest one. $\mathrm{SiO}_{2}$ generally increased and $\mathrm{MgO}, \mathrm{Cr}$, and $\mathrm{Ni}$ contents significantly decreased with younger age (Fig. 11). Therefore, in the basanitic suites, the eruptive order is IM31B $\approx$ IM32 $(\sim 21 \mathrm{Ma})>\operatorname{IM} 01(\sim 5 \mathrm{Ma})>\operatorname{IM} 30 \approx \operatorname{IM} 29(\sim 1 \mathrm{Ma})$, indicating that the magma became more evolved. Geochemical and isotopic variations among basanitic samples show that the sequence of magmatism represents increasing fractional crystallization of an evolved magma. Nevertheless, the magma source of the basanite retained isotopic heterogeneity due to its different proportions or heterogeneous sources of contributions (i.e., lithospheric mantle component; see next section).

\subsubsection{Tholeiites and Basanites: Products of Astheno- spheric-Lithospheric Mantle Interactions}

Early Tertiary to Quaternary basaltic lavas occurred at many locations in eastern China, extending from $\mathrm{Wu}$ dalianchi-Erkeshan-Keluo (WEK) in the north to the Leizhou Peninsula and the northern part of Hainan Island in the south. In several volcanic areas of eastern China there are similar $\mathrm{Sr}-\mathrm{Nd}-\mathrm{Pb}$ isotopic ratios between alkali basalts and tholeiites. For example, such trends occur in the Leiqiong and Zhejiang-Fujian regions (Ho et al. 2000, 2003). But many studies of tholeiites and alkali basalts from North China showed that both types of rocks are isotopically heterogeneous (e.g., Hannuoba, Song et al. 1990; Datong, Xu et al. 2005; Fanhsi, Tang et al. 2006), and their generation required the involvement of varying proportions of different mantle components.

As discussed above, three basaltic rock types (basanites, alkali olivine basalts, and tholeiites) in the JVF showed distinctive chemical attributes, which could not have 

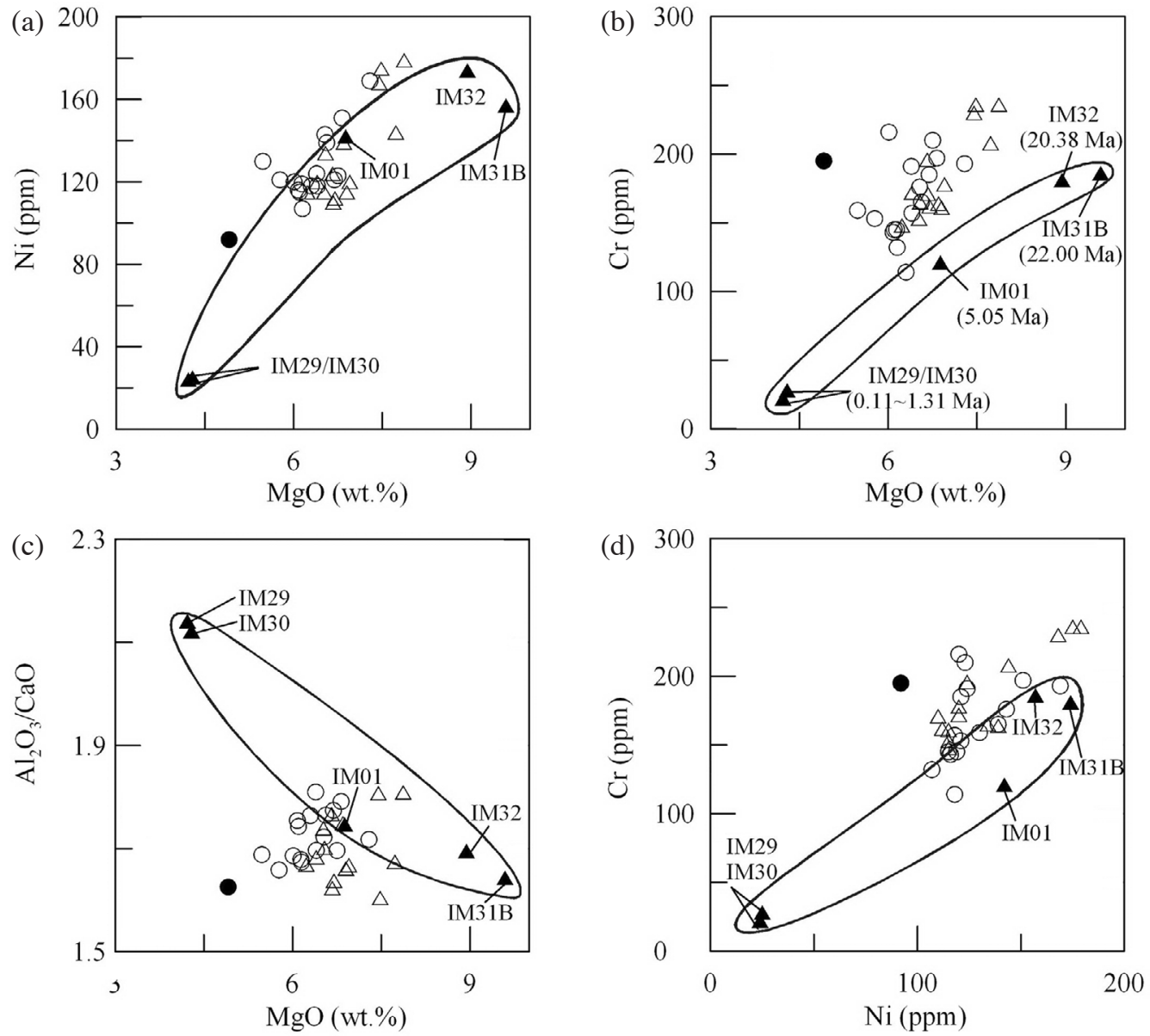

Fig. 11. (a) $\mathrm{Ni}$ vs. $\mathrm{MgO}$; (b) $\mathrm{Cr}$ vs. $\mathrm{MgO}$; (c) $\mathrm{Al}_{2} \mathrm{O}_{3} / \mathrm{CaO}$ vs. $\mathrm{MgO}$; and (d) $\mathrm{Cr}$ vs. Ni plots for the Jining basalts. The content of $\mathrm{MgO}$ decreases, whereas the $\mathrm{Ni}$ and $\mathrm{Cr}$ contents decrease and $\mathrm{Al}_{2} \mathrm{O}_{3} / \mathrm{CaO}$ ratio increases with decreasing age [given in Ma, as shown in (b)] in the basanites. Symbols are the same as those described in Fig. 3.

evolved from each other at shallow levels by magmatic differentiation. The Jining basanites and tholeiites have similar Sr-Nd isotopic compositions to those of Fanhsi high-Ti and low-Ti basalts, respectively (Fig. 6). But basanites generally overlap with the other western North China basalts in terms of $\mathrm{Sr}-\mathrm{Nd}-\mathrm{Pb}$ isotope ratios, while the tholeiites and alkali olivine basalt differ from basanites by their higher ${ }^{87} \mathrm{Sr} /{ }^{86} \mathrm{Sr}$, lower ${ }^{143} \mathrm{Nd} /{ }^{144} \mathrm{Nd}$, and less-radiogenic $\mathrm{Pb}$ isotope ratios, showing the imprints of lithospheric mantle components. In general, Hannuoba tholeiites have higher ${ }^{87} \mathrm{Sr} /{ }^{86} \mathrm{Sr}$ and lower ${ }^{143} \mathrm{Nd} /{ }^{144} \mathrm{Nd}$ ratios than the associated alkali basalts (Fig. 6). Meanwhile, tholeiitic rocks from the JVF have higher $\mathrm{Sr}$ and lower $\mathrm{Nd}$ and $\mathrm{Pb}$ isotopic ratios than those of Hannuoba tholeiites, and close to the range of Wudalianchi potassic volcanic rocks (Figs. 6, 7). Such variations in isotopic ratios of Jining basalts would require a compositionally heterogeneous source or mixing of compositionally different sources, because isotopic ratios are unaffected by partial melting and fractional crystallization.

Because the potassic volcanic rocks in the WEK have high ${ }^{87} \mathrm{Sr} /{ }^{86} \mathrm{Sr}$, low ${ }^{143} \mathrm{Nd} /{ }^{144} \mathrm{Nd}$, and low ${ }^{206} \mathrm{~Pb} /{ }^{204} \mathrm{~Pb}$ ratios
(Figs. 6, 7), Basu et al. (1991) argued that crustal assimilation was minimal, and the isotopic signatures of these volcanic rocks were inherited from the subcontinental mantle. Zhang et al. (1998c) and Zou et al. (2003) further demonstrated that these magmas were predominantly generated from a phlogopite-bearing garnet peridotite source located in the lower part of the subcontinental lithospheric mantle, which was metasomatized during Proterozoic time (Zhang et al. 1995). For most Cenozoic volcanic rocks of eastern China, basaltic magmas may have resulted from mixing of different proportions of depleted mid-oceanic ridge basalts (DMM or MORB) or FOZO components from the asthenospheric mantle with an enriched mantle component (EM1 or EM2) from the lithospheric mantle (e.g., Chung et al. 1994; Ho et al. 2008; Yan and Zhao 2008). For example, in the Hannuoba region, the alkali basalts have ${ }^{87} \mathrm{Sr} /{ }^{86} \mathrm{Sr}$ ratios of $0.7038 \sim 0.7040$, while tholeiites have values in the range of $0.7040 \sim 0.7048$ (Song et al. 1990). These differences were thought to be due to partial melting of different source materials. Xu et al. (2005) also concluded that the mantle source of Datong tholeiitic basalts might have been contaminated 
by the lithospheric mantle with an EM1 signature. In addition, it was noted by previous workers (e.g., Song et al. 1990; Basu et al. 1991) that the EM1 component did not accurately serve as the end-member for eastern Chinese lavas, especially for WEK potassic volcanic rocks. Therefore, Chen et al. (2007) further interpreted the $\mathrm{Sr}-\mathrm{Nd}-\mathrm{Pb}$ isotopic data of potassic basalts from NE China as being the result of mixing between a FOZO end-member and a LoMu endmember.
Lavas that erupted within the JVF are mixtures derived from depleted mantle rocks similar to the Indian Ocean MORB (DMM component) and less-depleted or enriched source rocks represented by an EM1 component. This conclusion can be clearly derived from the ${ }^{87} \mathrm{Sr} /{ }^{86} \mathrm{Sr}$ and ${ }^{143} \mathrm{Nd} /{ }^{144} \mathrm{Nd}$ vs. ${ }^{206} \mathrm{~Pb} / 204 \mathrm{~Pb}$ correlations of the basaltic rocks (Fig. 12). An EM1 component was commonly suggested to be derived from the continental lithosphere mantle (e.g., Song et al. 1990) and originated from ancient metasomatic

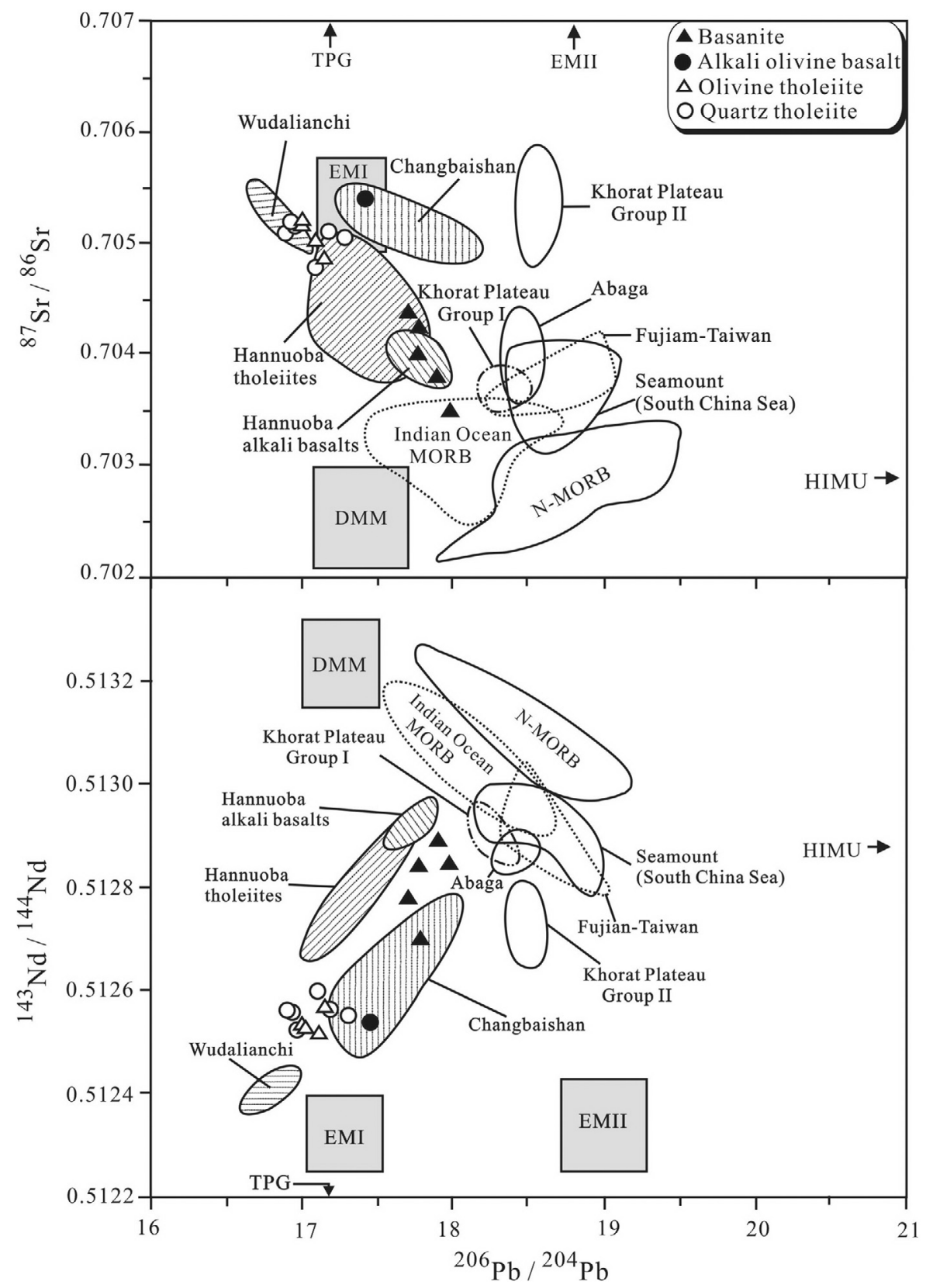

Fig. $12 .{ }^{87} \mathrm{Sr} /{ }^{86} \mathrm{Sr}$ and ${ }^{143} \mathrm{Nd} /{ }^{144} \mathrm{Nd}$ vs. ${ }^{206} \mathrm{~Pb} / 204 \mathrm{~Pb}$ diagrams for the Jining basalts. The fields for the Wudalianchi, Changbaishan, Hannuoba, Abaga, Fujian-Taiwan, South China Sea seamount, Indian Ocean mid-ocean ridge basalt (MORB), and N-type (N)-MORB are shown for comparison. Wudalianchi and Changbaishan are from Basu et al. (1991); other data sources are the same as those in Fig. 7. Geochemical compositions of depleted MORB mantle (DMM), enriched mantle of type 1 (EM1), EM2, and two-pyroxene granulite (TPG) are adopted from Zindler and Hart (1986) and Zhang et al. (1998b). 
event(s) (e.g., Tatsumoto and Nakamura 1991). Therefore, we suggest that magmas of Jining tholeiites were predominantly derived from an isotopically enriched subcontinental lithosphere component.

Lithospheric contamination of the asthenospheric mantle can occur through ancient subduction processes (Hofmann 1988), lithospheric delamination (McKenzie and O'Nions 1995), or melts interacting with the lithospheric mantle before erupting to the surface (Class and Goldstein 1997). It is generally believed that the NCC experienced widespread thermotectonic reactivations during late Mesozoic and Cenozoic times (Griffin et al. 1998; Xu et al. 2001; Menzies et al. 2007). Based on isotopic features, there were significant interactions between the asthenosphere and lithospheric mantle in the western NCC. Therefore, a "plumpudding mixing model" suggested by Morris and Hart (1983) may be useful in considering the petrogenesis of most basaltic rocks in the JVF. The Early Oligocene Jining basalts, which occurred through voluminous partial melting of thermal-eroded continental lithospheric materials, are mainly quartz- and olivine-normative tholeiites. In the late Tertiary-Quaternary, the basalts were mainly derived from an asthenospheric source; therefore, basanites progressively became the dominant rock type.

\subsubsection{Alkali Olivine Basalts: Product of Basanitic Mag- ma Mixing with Two-Pyroxene Granulite (TPG)}

The very striking features of the major and trace element data are that no significant correlations could be found between alkali olivine basalts and basanites or tholeiites. Although the alkali olivine basalts had similar abundances of $\mathrm{Al}_{2} \mathrm{O}_{3}, \mathrm{CaO}, \mathrm{Na}_{2} \mathrm{O}$, and $\mathrm{Cr}$, they always contained higher incompatible elements (e.g., $\mathrm{Ba}, \mathrm{Th}, \mathrm{Nb}$, and $\mathrm{Sr}$ ) and $\mathrm{K}_{2} \mathrm{O}$ and lower $\mathrm{MgO}, \Sigma \mathrm{FeO}, \mathrm{MnO}, \mathrm{Sc}$, and $\mathrm{Ni}$ abundances than the tholeiites at a given $\mathrm{SiO}_{2}$ content (Figs. 3, 5). In addition, alkali olivine basalts also have higher $\mathrm{SiO}_{2}$ and lower $\Sigma \mathrm{FeO}$ contents compared to the basanites. In Ne-OlDi-Hy-Qz tetrahedron and major element oxides vs. $\mathrm{SiO}_{2}$ plots (Figs. 2, 3), therefore, the alkali olivine basalts always fall off the trends established by tholeiitic or basanitic rock samples.

Alkali olivine basalts, with enriched mantle characteristics, have higher ${ }^{87} \mathrm{Sr} /{ }^{86} \mathrm{Sr}$ and lower ${ }^{143} \mathrm{Nd} /{ }^{144} \mathrm{Nd}$, ${ }^{206} \mathrm{~Pb} /{ }^{204} \mathrm{~Pb}$, and ${ }^{207} \mathrm{~Pb} /{ }^{204} \mathrm{~Pb}$ ratios than the basanites (Figs. 6, 7), which preclude their derivation from a compositionally and isotopically homogeneous source. On the other hand, alkali olivine basalts have apparently similar ${ }^{143} \mathrm{Nd} /{ }^{144} \mathrm{Nd}$ and ${ }^{207} \mathrm{~Pb} /{ }^{204} \mathrm{~Pb}$ but slightly higher ${ }^{87} \mathrm{Sr} /{ }^{86} \mathrm{Sr},{ }^{206} \mathrm{~Pb} /{ }^{204} \mathrm{~Pb}$, and ${ }^{208} \mathrm{~Pb} /{ }^{204} \mathrm{~Pb}$ ratios compared to the tholeiites. These compositional differences may be related to the chemical characteristics of the magma sources. Indeed, to our knowledge, this type of basaltic rock has not been reported in the NCC before.
In view of the geochemical characteristics of the alkali olivine basaltic magmas, it is unlikely that they were generated by assimilation of upper continental crustal materials. For example, the contents of $\mathrm{CaO}(8.97 \mathrm{wt} \%)$ and $\mathrm{Cr}$ (195 ppm) of this rock sample are much higher than those of the upper continental crust $(3.59 \mathrm{wt} \% \mathrm{CaO}$ and $92 \mathrm{ppm}$ $\mathrm{Cr}$, Rudnick and Gao 2003). Although there are no apparent mantle and lower-crust xenoliths in the Jining basalts, the alkali-rich lavas of the Hannuoba area some $100 \mathrm{~km}$ to the east host abundant deep-seated xenoliths. The xenoliths vary from ultramafic to mafic to felsic with peridotites being dominant, pyroxenites and mafic granulites subordinate, and felsic granulites rare (Zhang et al. 1998a). Petrographically, the granulite xenoliths have diverse mineral constituents and can be subdivided into three types: felsic, noritic, and TPG. TPG is composed of clinopyroxene + orthopyroxene + plagioclase \pm biotite \pm K-feldspar, which may be widely distributed in the lower crust beneath the Hannuoba region (Zhang et al. 1998a). On the basis of detailed isotopic studies of TPG xenoliths, Zhang et al. (1998a, b) reported a very wide range in ${ }^{87} \mathrm{Sr} /{ }^{86} \mathrm{Sr}(0.7059 \sim 0.7086$; average of $0.7072),{ }^{143} \mathrm{Nd} /{ }^{144} \mathrm{Nd}(0.5117 \sim 0.5122$; average of 0.5119$)$, ${ }^{206} \mathrm{~Pb} /{ }^{204} \mathrm{~Pb}(16.5 \sim 17.9$; average of 17.2$),{ }^{207} \mathrm{~Pb} /{ }^{204} \mathrm{~Pb}(15.2 \sim$ 15.7; average of 15.4$)$, and ${ }^{208} \mathrm{~Pb} /{ }^{204} \mathrm{~Pb}(36.5 \sim 38.3$; average of 37.4) ratios, therefore, they suggested that the lower crust beneath the Hannuoba area is highly chemically heterogeneous.

In the ${ }^{87} \mathrm{Sr} /{ }^{86} \mathrm{Sr}$ and ${ }^{143} \mathrm{Nd} /{ }^{144} \mathrm{Nd}$ vs. ${ }^{206} \mathrm{~Pb} /{ }^{204} \mathrm{~Pb}$ diagrams (Fig. 12), the alkali olivine basalts were plotted between basanites and the average compositions of TPG, indicating that at least three end-members would have been involved in the genesis of alkali olivine basalts: i.e., EM1, DMM, and TPG. The integration of major and trace element compositions showed a similar mixing trend (data not shown). Based on the observations, combined with a lower than average $\mathrm{Nb} / \mathrm{U}$ value $(47 \pm 10$, Hofmann et al. 1986) of OIBs and MORBs, we suggest that the alkali olivine basalts could have been generated by basanitic magma interacting with the mafic lower crust (i.e., two-pyroxene granulite) en route to the surface.

\subsection{Geodynamic Implications}

K-Ar dating results of Jining basaltic rocks showed a wide range of ages from nearly 36 to $<0.2 \mathrm{Ma}$, indicating that magmatic activities in the JVF lasted from the early Oligocene to the late Pleistocene. The content of $\mathrm{SiO}_{2}$ decreased, whereas the incompatible elements, e.g., $\mathrm{K}_{2} \mathrm{O}$, Th, and $\mathrm{Ba}$ contents, and the $(\mathrm{La} / \mathrm{Yb})_{\mathrm{N}}$ ratio, increased with younger age of the basaltic rocks in the JVF. These compositional changes may reflect evolutionary trends of the magma from a tholeiitic to an alkalic character. Additional evidence of the evolutionary trend of the Jining basaltic magmas was provided by the combination of the $\mathrm{Sr}-\mathrm{Nd}-\mathrm{Pb}$ isotope 
ratios (Figs. 6, 7). The lavas that erupted within the JVF are characterized by significant variations in $\mathrm{Sr}-\mathrm{Nd}-\mathrm{Pb}$ isotopic compositions with younger ages towards more MORB-like isotopic signatures. The above two lines of evidence clearly show that a relationship may exist between volcanism and geodynamic evolution in the JVF for a period from the early Tertiary to the Quaternary.

From the K-Ar dates and spatial distribution of the basaltic rocks, a general trend of Cenozoic volcano-tectonic evolution of the JVF is proposed as follows (Fig. 13). At $36 \sim 33 \mathrm{Ma}$, mixing processes may have occurred during a rifting event in the JVF. Strong thermal effects from the ascending asthenosphere caused thinning of the continental lithosphere and melting of thermal-eroded materials, producing large amounts of tholeiitic lavas with high and restricted ranges of $\mathrm{Sr}$ isotope ratios. At $23 \sim 9 \mathrm{Ma}$, interactions between the upwelling asthenosphere and lithosphere may have weakened. According to geochemical data, transport of materials from the asthenosphere mantle gradually played an important role. After $5 \mathrm{Ma}$, the upwelling asthenospheric mantle became the predominant magma source. Sparse volcanic activities occurred and produced small amounts of basanitic lavas or pyroclastic rocks that were mainly emplaced on the northern and eastern JVF margins. It should be noted that tholeiites occurred in the older lava flows and formed a large lava platform, whereas alkalic samples were collected from smaller and younger lava flows or volcanic cones. Therefore, the volcanic activity in the JVF was characterized by a gradual transition from flows along fissures to eruptive centers.

The Cenozoic basaltic rocks form four mainly eruptive areas in the western NCC: the Jining, Fanhsi, Hannuoba, and Datong volcanic fields (Fig. 1). The Fanhsi basalts were previously dated to the early Oligocene $(40.4 \sim 37.1 \mathrm{Ma}$, Wang et al. 1989) and were thought to represent the earliest volcanic activity in the western NCC. In fact, most basaltic rocks (19 data) analyzed by Liu et al. (1992b), Tang et al. (2006), and Ho (unpublished data) in the Fanhsi volcanic field erupted at ages younger than $30 \mathrm{Ma}$. Therefore, Tang et al. (2006) suggested that the Fanhsi basalts have similar ages as the Hannuoba basalts. In the present study, new $\mathrm{K}$-Ar data indicate that the main episode of tholeiitic volcanism in JVF, dated $36 \sim 33 \mathrm{Ma}$, was roughly earlier in occurrence than most of the Fanhsi alkali basalts.

The spatial distribution of volcanics of different ages can be correlated with the order of migration of the volcanic foci. Based on the systematic K-Ar geochronology studies of the western NCC (Luo and Chen 1990; Chen et al. 1992; Liu et al. 1992b; Tang et al. 2006; Ho, unpublished data; this work), we established the time span of the volcanic activity for each volcanic field as $36.4 \sim 0.11 \mathrm{Ma}$ for the Jining, $30.9 \sim 23.7$ Ma for the Fanhsi, $26.8 \sim 4.4$ Ma for the Hannuoba, and $0.88 \sim 0.23$ Ma for the Datong. Therefore, Cenozoic principal volcanic activity in the western NCC is believed to have begun in the Jining volcanic fields, and then migrated southeastward to Fanhsi and northeastward to Hannuoba. Finally, one eruption center migrated southward to the Xiyang-Pingding $(8 \sim 7 \mathrm{Ma})$ and Zouquan $(\sim 5.6 \mathrm{Ma})$ areas (i.e., near the DTGL), and another eruption center was localized in the Datong volcanic field in the Quaternary.

Fanhsi volcanics are mainly alkaline in composition (Xu et al. 2004); Hannuoba volcanic rocks are alkali basalts at the bottom and grade to intercalations of alkali basalts and tholeiites at the top (Zhi et al. 1990). Basalts from the western and eastern Datong volcanic field have different mineralogies whereby the former is dominated by alkalic lava flows, while the basaltic rocks in the latter are tholeiitic (Xu et al. 2005). Based on the geochemistry of Cenozoic basalts from the above three volcanic fields, Xu et al. (2004) concluded that progressive lithospheric thinning occurred in the western NCC during the Cenozoic. As noted previously, similar to many other volcanic regions in eastern Asia, for instance, in the Leiqiong (Ho et al. 2000), and XinchangShengxian areas (Ho et al. 2003), older basalts are generally tholeiites and younger basalts are increasingly alkalic as also occurred in the JVF. Tholeiitic rocks may be related to an early phase of rifting, and basanitic rocks to the late stage of rifting systems. The generation of voluminous igneous melts clearly reflects major thermal events. The JVF is the earliest and largest Cenozoic volcanic province in the western NCC. Therefore, we suggest that the largest degree of thinning/erosion of lithospheric mantle thermo-mechanical processes with upwelling of the asthenosphere beneath the western NCC may have occurred during the early Oligocene.

\section{CONCLUSION}

The following conclusion can be reached from the integrated $\mathrm{K}-\mathrm{Ar}$ age, elemental, and $\mathrm{Sr}-\mathrm{Nd}-\mathrm{Pb}$ isotopic studies of Jining basalts presented in this study.

(1) The voluminous and widespread Jining basaltic lavas along the southern margin of the Mongolian Plateau predominantly consist of quartz tholeiites and olivine tholeiites with subordinate basanites and rare alkali olivine basalts. All samples of the JVF analyzed in the present study are high- $\mathrm{Ti}\left(\mathrm{Ti} / \mathrm{Y}>462, \mathrm{TiO}_{2} \approx 1.5 \sim\right.$ 2.9 wt \%) and sodic with $\mathrm{Na}_{2} \mathrm{O} / \mathrm{K}_{2} \mathrm{O}>1$ and $\mathrm{SiO}_{2}$ contents ranging $44 \sim 54 \mathrm{wt} \%$. Basanites with significantly variable $\mathrm{MgO}, \mathrm{Ni}$, and $\mathrm{Cr}$ contents and $\mathrm{Al}_{2} \mathrm{O}_{3} / \mathrm{CaO}$ ratios might have experienced fractional crystallization of olivine and clinopyroxene. However, $\mathrm{MgO}$ and $\Sigma \mathrm{FeO}$ contents of tholeiites display a weak trend with $\mathrm{SiO}_{2}$, suggesting that small amounts of olivine fractional crystallization may have occurred after the formation of the initial melt.

(2) Jining tholeiites show moderate incompatible element 
(a)

$36 \sim 33 \mathrm{Ma}$

Jining/Tholeiites

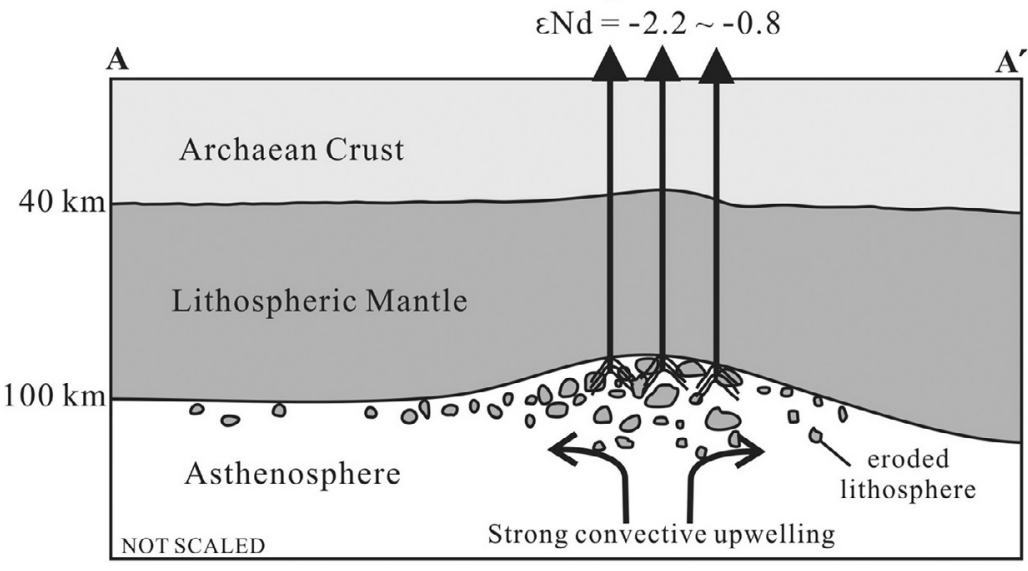

(b)

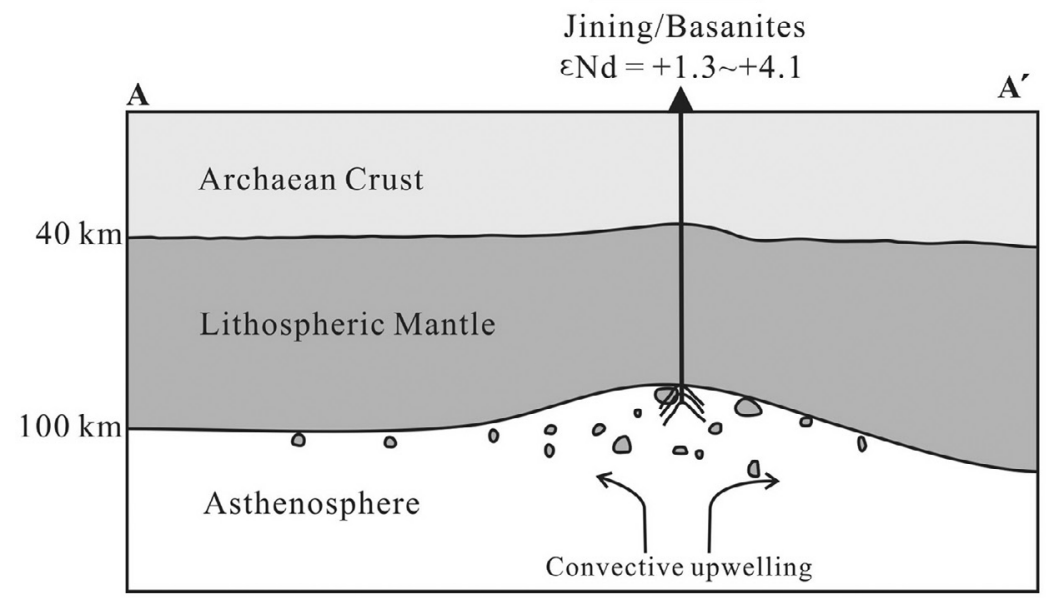

(c)

$$
1.3 \sim 0.1 \mathrm{Ma}
$$

Qahar Youyi Houqi/Basanites

$\varepsilon N d=+2.8 \sim+3.9$

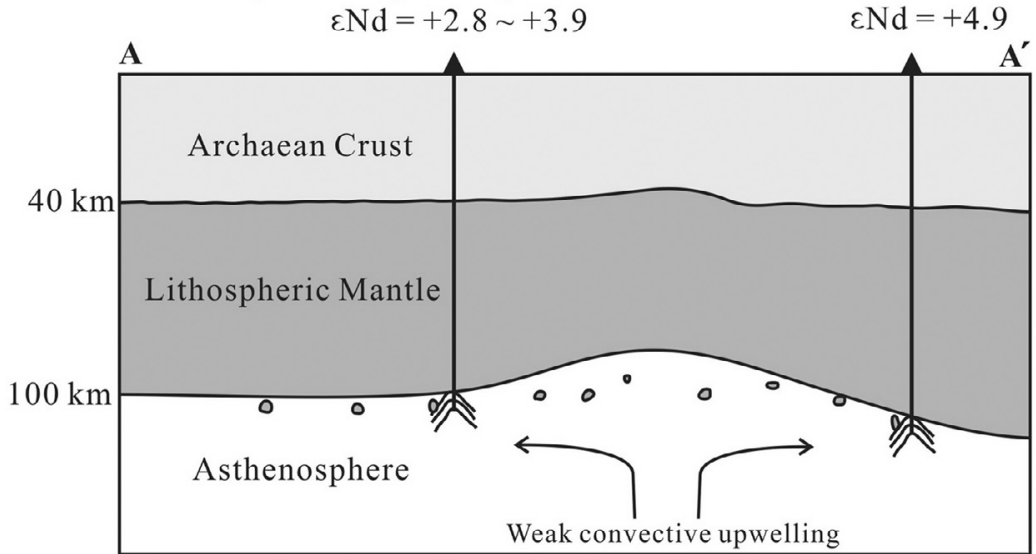

Fig. 13. Schematic model illustrating the generation of Cenozoic basalts in the Jining volcanic field (JVF). The magmas were produced from partial melting of the asthenosphere and interaction with the lithospheric mantle through thermo-mechanical processes. (a) At $36 \sim 33 \mathrm{Ma}$, basalts occurred voluminously in the JVF, which were mainly quartz- and olivine-normative tholeiites; (b) at $22 \sim 20 \mathrm{Ma}$ and (c) after 5 Ma, basalt compositions were consistent with a dominant asthenospheric source for the basanites. Cross-section of the lithosphere constructed on the basis of data by Ma (1989) and Xu et al. (2004). Location of the cross-section is shown in Fig. 1. 
enrichment and have a limited range of $\mathrm{Sr}-\mathrm{Nd}$ isotopic compositions more enriched than the Bulk Silicate Earth reservoir; in contrast, Jining basanites display an OIBlike incompatible element pattern and have a wider range of $\mathrm{Sr}-\mathrm{Nd}$ isotopic compositions with depleted characteristics similar to those of Hannuoba basaltic rocks. The distinct differences in isotopic ratios between both rock types suggest that they were not derived from a common mantle reservoir, and mixing processes may have played an important role in the genesis of Jining basaltic rocks. Based on a detailed $\mathrm{Sr}-\mathrm{Nd}-\mathrm{Pb}$ isotope study, we can demonstrate that the tholeiites were derived from a mixed DMM-EM1 source dominated by lithospheric mantle components, whereas basanites were derived from a source region containing a higher ratio of the underlying asthenospheric mantle component.

(3) Rare alkali olivine basalts occur in the JVF but have markedly different geochemical characteristics from the tholeiites and basanites. The highest Sr-isotopic ratio and enrichment of trace element contents indicate that this type of basalt underwent more-complicated genetic processes. According to the elemental and $\mathrm{Sr}-\mathrm{Nd}-\mathrm{Pb}$ isotopic data, interactions of basanitic melts with the lower crust may explain the genesis of alkali olivine basaltic magma.

(4) $\mathrm{K}$-Ar dating results of these basaltic rocks yielded ages of $36 \sim 0.1 \mathrm{Ma}$ and with obvious relationships among the eruptive times, chemical compositions, and occurrence types. Large-scale tholeiitic eruptions took place in the early Oligocene, mainly consisting of voluminous lava flows through extensional fissures, in contrast to small-volume basanites, which mainly occur in the eastern to northern margins of the JVF and were predominant products of central volcanoes during the late Tertiary-Quaternary period. Considered as a whole, the main volcanic activities in the JVF began earlier and lasted longer than those of the other Cenozoic volcanic fields in the western NCC.

Acknowledgements We are grateful to F. K. Chen of the Laboratory for Radiogenic Isotope Geochemistry, Institute of Geology and Geophysics, Chinese Academy of Sciences and B. S. Wang of the Earth Dynamic System Research Center, NCKU for their assistance with Pb-isotope measurement. D. M. Lee is kindly thanked for his help with dating work in the K-Ar laboratory at State Key Laboratory of Earthquake Dynamics, Institute of Geology, China Earthquake Administration. We thank Y. J. Lai for operating the ICP-MS and M. H. Huang for preparing the figures. Penetrating reviews by K. L. Wang, J. B. Lan and S. L. Chung significantly improved the clarity and consistency of the manuscript, while Associate Editor T. Y. F. Yang is thanked for valuable comments and editorial handling. This research was funded by a grant from the National Science
Council, Taiwan, ROC (NSC95-2116-M-178-002) to K. S. Ho. Field work was supported by the National Museum of Natural Science, Taiwan.

\section{REFERENCES}

Basu, A. R., J. Wang, W. Huang, G. Xie, and M. Tatsumoto, 1991: Major element, REE, and $\mathrm{Pb}, \mathrm{Nd}$ and $\mathrm{Sr}$ isotopic geochemistry of Cenozoic volcanic rocks of eastern China: implications for their origin from suboceanictype mantle reservoirs. Earth Planet. Sci. Lett., 105, 149-169, doi: 10.1016/0012-821X(91)90127-4. [Link]

Bureau of Geology and Mineral Resources of Inner Mongolia Autonomous Region, 1991: Regional geology of Inner Mongolia Autonomous Region, Geological Publishing House, Beijing, 725 pp. (in Chinese)

Chen, F., E. Hegner, and W. Todt, 2000: Zircon ages and Nd isotopic and chemical compositions of orthogneisses from the Black Forest, Germany: Evidence for a Cambrian magmatic arc. Int. J. Earth Sci., 88, 791-802, doi: 10.1007/s005310050306. [Link]

Chen, W. J., D. M. Li, T. M. Dai, Z. P. Pu, R. X. Liu, Q. Li, J. Z. Shun, X. Wang, E. Jäger, A. J. Hurford, and H. R. Pfeifer, 1992: The K-Ar age and excess Ar of Quaternary basalt in Datong. In: Liu, R. X. (Ed.), The Age and Geochemistry of Cenozoic Volcanic Rock in China, Seismological Press, Beijing, 81-92. (in Chinese)

Chen, Y., Y. Zhang, D. Graham, S. Su, and J. Deng, 2007: Geochemistry of Cenozoic basalts and mantle xenoliths in Northeast China. Lithos, 96, 108-126, doi: 10.1016/j.lithos.2006.09.015. [Link]

Chih, C. S., 1988: The Study of Cenozoic Basalts and the Upper Mantle beneath Eastern China (Attachment: Kimberlites), China Geosciences University Press, Wuhan, 277 pp. (in Chinese)

Chung, S. L., S. S. Sun, K. Tu, C. H. Chen, and C. Y. Lee, 1994: Late Cenozoic basaltic volcanism around the Taiwan Strait, SE China: Product of lithosphere-asthenosphere interaction during continental extension. Chem. Geol., 112, 1-20, doi: 10.1016/0009-2541(94)90101-5. [Link]

Chung, S. L., T. F. Yang, S. J. Chen, C. H. Chen, T. Lee, and C. H. Chen, 1995: Sr-Nd isotope compositions of high-pressure megacrysts and a lherzite inclusion in alkali basalts from western Taiwan. J. Geol. Soc. China, 38, 15-24.

Class, C. and S. L. Goldstein, 1997: Plume-lithosphere interactions in the ocean basins: Constraints from the source mineralogy. Earth Planet. Sci. Lett., 150, 245260, doi: 10.1016/S0012-821X(97)00089-7. [Link]

Dupré, B. and C. J. Allègre, 1983: Pb-Sr isotope variation in Indian Ocean basalts and mixing phenomena. Nature, 303, 142-146, doi: 10.1038/303142a0. [Link] 
Glazner, A. F. and G. L. Farmer, 1992: Production of isotopic variability in continental basalts by cryptic crustal contamination. Science, 255, 72-74, doi: 10.1126/science.255.5040.72. [Link]

Griffin, W. L., A. D. Zhang, S. Y. O'Reilly, and C. G. Ryan, 1998: Phanerozoic evolution of the lithosphere beneath the Sino-Korean Craton. In: Flower, M. F. J., S. L. Chung, C. H. Lo, and T. Y. Lee (Eds.), Mantle Dynamics and Plate Interactions in East Asia, Am. Geophys. Union Geodyn. Ser., 27, 107-126.

Han, B. F., S. G. Wang, and H. Kagami, 1999: Trace element and $\mathrm{Nd}-\mathrm{Sr}$ isotopic constraints on origin of the Chifeng flood basalts, North China. Chem. Geol., 155, 187-199, doi: 10.1016/S0009-2541(98)00172-7. [Link]

Hart, S. R., 1984: A large-scale isotope anomaly in the Southern Hemisphere mantle. Nature, 309, 753-757, doi: 10.1038/309753a0. [Link]

Hart, S. R. and C. J. Allègre, 1980: Trace element constraints on magma genesis. In: Hargraves, R. B. (Ed.), Physics of Magmatic Processes, Princeton Univ. Press, New Jersey, 121-139.

Hart, S. R., D. C. Gerlach, and W. M. White, 1986: A possible new $\mathrm{Sr}-\mathrm{Nd}-\mathrm{Pb}$ mantle array and consequences for mantle mixing. Geochim. Cosmochim. Acta, 50, 15511557, doi: 10.1016/0016-7037(86)90329-7. [Link]

Ho, K. S., J. C. Chen, and W. S. Juang, 2000: Geochronology and geochemistry of late Cenozoic basalts from the Leiqiong area, southern China. J. Asian Earth Sci., 18, 307-324, doi: 10.1016/S1367-9120(99)00059-0. [Link]

Ho, K. S., J. C. Chen, C. H. Lo, and H. L. Zhao, 2003: ${ }^{40} \mathrm{Ar}-$ ${ }^{39} \mathrm{Ar}$ dating and geochemical characteristics of late $\mathrm{Ce}$ nozoic basaltic rocks from the Zhejiang-Fujian region, SE China: Eruption ages, magma evolution and petrogenesis. Chem. Geol., 197, 287-318, doi: 10.1016/S0 009-2541(02)00399-6. [Link]

Ho, K. S., Y. Liu, J. C. Chen, and H. J. Yang, 2008: Elemental and $\mathrm{Sr}-\mathrm{Nd}-\mathrm{Pb}$ isotopic compositions of late Cenozoic Abaga basalts, Inner Mongolia: Implications for petrogenesis and mantle process. Geochem. J., 42, 339-357.

Hofmann, A. W., 1988: Chemical differentiation of the Earth: The relationship between mantle, continental crust, and oceanic crust. Earth Planet. Sci. Lett., 90, 297-314, doi: 10.1016/0012-821X(88)90132-X. [Link]

Hofmann, A. W., K. P. Jochum, M. Seufert, and W. M. White, 1986: $\mathrm{Nb}$ and $\mathrm{Pb}$ in oceanic basalts: New constraints on mantle evolution. Earth Planet. Sci. Lett., 79, 33-45, doi: 10.1016/0012-821X(86)90038-5. [Link]

Liu, D. Y., A. P. Nutman, W. Compston, J. S. Wu, and Q. H. Shen, 1992a: Remnants of $\geqq 3800$ Ma crust in the Chinese part of the Sino-Korean craton. Geology, 20, 339-342, doi: 10.1130/0091-7613(1992)020<0339:RO MCIT>2.3.CO;2. [Link]
Liu, R. X., W. J. Chen, J. Z. Sun, and D. M. Li, 1992b: The $\mathrm{K}-\mathrm{Ar}$ age and tectonic environment of Cenozoic rock in China. In: Liu, R. X. (Ed.), The Age and Geochemistry of Cenozoic Volcanic Rock in China, Seismological Press, Beijing, 1-43. (in Chinese)

Luo, X. Q. and Q. T. Chen, 1990: Preliminary study on geochronology for Cenozoic basalts from Inner Mongolia. Acta Petrol. Mineral., 9, 37-46. (in Chinese)

Ma, X., 1989: Atlas of Active Faults in China, Seismological Press, Beijing, 120 pp.

Mahoney, J. J., J. H. Natland, W. M. White, R. Poreda, S. H. Bloomer, R. L. Fisher, and A. N. Baxter, 1989: Isotopic and geochemical provinces of the western Indian Ocean spreading centers. J. Geophys. Res., 94, 40334052.

McKenzie, D. and R. K. O’Nions, 1995: The source regions of ocean island basalts. J. Petrol., 36, 133-159.

Menzies, M., Y. Xu, H. Zhang, and W. Fan, 2007: Integration of geology, geophysics and geochemistry: A key to understanding the North China Craton. Lithos, 96, 1-21, doi: 10.1016/j.lithos.2006.09.008. [Link]

Morris, J. D. and S. R. Hart, 1983: Isotopic and incompatible element constraints on the genesis of island arc volcanics from Cold Bay and Amak Island, Aleutians, and implications for mantle structure. Geochim. Cosmochim. Acta, 47, 2015-2030, doi: 10.1016/0016-7037 (83)90217-X. [Link]

Rudnick, R. L. and S. Gao, 2003: Composition of the continental crust. In: Rudnick, R. L. (Ed.), The Crust., Vol. 3., Treatise on Geochemistry, Holland, H. D. and K. K. Turekian (Eds.), Elsevier-Pergamon, Oxford, 1-64, doi: 10.1016/B0-08-043751-6/03016-4. [Link]

Song, Y., F. A. Frey, and X. Zhi, 1990: Isotopic characteristics of Hannuoba basalts, eastern China: Implications for their petrogenesis and the composition of subcontinental mantle. Chem. Geol., 85, 35-52, doi: 10.1016/0009-2541(90)90102-D. [Link]

Steiger, R. H. and E. Jäger, 1977: Subcommission on geochronology: Convention on the use of decay constants in geo- and cosmochronology. Earth Planet. Sci. Lett., 36, 359-362, doi: 10.1016/0012-821X(77)9006 0-7. [Link]

Sun, S. S. and W. F. McDonough, 1989: Chemical and isotopic systematics of oceanic basalts: Implications for mantle composition and processes. J. Geol. Soc. London, Spec. Publ., 42, 313-345, doi: 10.1144/GSL. SP.1989.042.01.19. [Link]

Tang, Y. J., H. F. Zhang, and J. F. Ying, 2006: Asthenosphere-lithospheric mantle interaction in an extensional regime: Implication from the geochemistry of $\mathrm{Ce}$ nozoic basalts from Taihang Mountains, North China Craton. Chem. Geol., 233, 309-327, doi: 10.1016/j.che mgeo.2006.03.013. [Link]

Tang, Y. J., H. F. Zhang, J. F. Ying, J. Zhang, and X. M. Liu, 
2008: Refertilization of ancient lithospheric mantle beneath the central North China Craton: Evidence from petrology and geochemistry of peridotite xenoliths. $\mathrm{Li}$ thos, 101, 435-452, doi: 10.1016/j.lithos.2007.09.006. [Link]

Tatsumoto, M. and Y. Nakamura, 1991: DUPAL anomaly in the Sea of Japan: $\mathrm{Pb}, \mathrm{Nd}$, and $\mathrm{Sr}$ isotopic variations at the eastern Eurasian continental margin. Geochim. Cosmochim. Acta, 55, 3697-3708, doi: 10.1016/00167037(91)90068-G. [Link]

Tu, K., M. F. J. Flower, R. W. Carlson, G. H. Xie, C. Y. Chen, and M. Zhang, 1992: Magmatism in the South China Basin: 1. Isotopic and trace-element evidence for an endogenous Dupal mantle component. Chem. Geol., 97, 47-63, doi: 10.1016/0009-2541(92)90135R. [Link]

Wang, H., X. Yang, B. Zhu, S. Fan, and T. Dai, 1989: K-Ar geochronology and evolution of Cenozoic volcanic rocks in eastern China. Chin. J. Geochem., 8, 1-14, doi: 10.1007/BF02842210. [Link]

Wilson, M., 1989: Igneous Petrogenesis, Harper Collins Academic, London, $466 \mathrm{pp}$.

Xie, G. H., K. Tu, J. W. Wang, M. Zhang, and M. F. J. Flower, 1989: Lead isotopic compositions of Cenozoic basalts from eastern China: Geographical control and genetic significance. Chin. Sci. Bull., 34, 20552060.

Xu, Y. G., 2001: Thermo-tectonic destruction of the Archaean lithospheric keel beneath the Sino-Korean Craton in China: Evidence, timing and mechanism. Phys. Chem. Earth, Part A Solid Earth Geod., 26, 747-757, doi: 10.1016/S1464-1895(01)00124-7. [Link]

Xu, Y. G., S. L. Chung, J. Ma, and L. Shi, 2004: Contrasting Cenozoic lithospheric evolution and architecture in the western and eastern Sino-Korean Craton: Constraints from geochemistry of basalts and mantle xenoliths. $J$. Geol., 112, 593-605, doi: 10.1086/422668. [Link]

Xu, Y. G., J. L. Ma, F. A. Frey, M. D. Feigenson, and J. F. Liu, 2005: Role of lithosphere-asthenosphere interaction in the genesis of Quaternary alkali and tholeiitic basalts from Datong, western North China Craton. Chem. Geol., 224, 247-271, doi: 10.1016/j.chemgeo.20 05.08.004. [Link]

Yan, J. and J. X. Zhao, 2008: Cenozoic alkali basalts from Jingpohu, NE China: The role of lithosphere-asthenosphere interaction. J. Asian Earth Sci., 33, 106-121, doi: 10.1016/j.jseaes.2007.11.001. [Link]

Yoder, H. S. Jr. and C. E. Tilley, 1962: Origin of basalt magmas: An experimental study of natural and synthetic rock systems. J. Petrol., 3, 342-532.

Zhang, G. H., X. H. Zhou, M. Sun, S. H. Chen, and J. L. Feng, 1998a: Highly chemical heterogeneity in the lower crust and crust-mantle transitional zone: Geochemical evidences from granulite xenoliths in Han- nuoba basalts, Hebei Province. Chin. J. Geochemica, 27, 153-163. (in Chinese)

Zhang, G. H., X. H. Zhou, M. Sun, S. H. Chen, and J. L. Feng, 1998b: $\mathrm{Sr}, \mathrm{Nd}$ and $\mathrm{Pb}$ isotopic characteristics of granulite and pyroxenite xenoliths in Hannuoba basalts, Hebei Province, and their implications for geological processes. Acta Petrol. Sin., 14, 190-197. (in Chinese)

Zhang, J. J., Y. F. Zheng, and Z. F. Zhao, 2009: Geochemical evidence for interaction between oceanic crust and lithospheric mantle in the origin of Cenozoic continental basalts in east-central China. Lithos, 110, 305-326, doi: 10.1016/j.lithos.2009.01.006. [Link]

Zhang, M., P. Suddaby, R. N. Thompson, M. F. Thirlwall, and M. A. Menzies, 1995: Potassic volcanic rocks in NE China: Geochemical constraints on mantle source and magma genesis. J. Petrol., 36, 1275-1303.

Zhang, M., X. H. Zhou, and J. B. Zhang, 1998c: Nature of the lithospheric mantle beneath NE China: Evidence from potassic volcanic rocks and mantle xenoliths. In: Flower, M. F. J., S. L. Chung, C. H. Lo, and T. Y. Lee (Eds.), Mantle Dynamics and Plate Interactions in East Asia, Am. Geophys. Union Geodyn. Ser., 27, 197-219.

Zhang, Y. Q., J. L. Mercier, and P. Vergély, 1998d: Extension in the graben systems around the Ordos (China), and its contribution to the extrusion tectonics of south China with respect to Gobi-Mongolia. Tectonophysics, 285, 41-75, doi: 10.1016/S0040-1951(97)00170-4. [Link]

Zhao, G., S. A. Wilde, P. A. Cawood, and M. Sun, 2001: Archean blocks and their boundaries in the North China Craton: Lithological, geochemical, structural and $P-T$ path constraints and tectonic evolution. Precam. Res., 107, 45-73, doi: 10.1016/S0301-9268(00)001546. [Link]

Zheng, J., W. L. Griffin, S. Y. O'Reilly, F. Lu, C. Wang, M. Zhang, F. Wang, and H. Li, 2004: 3.6 Ga lower crust in central China: New evidence on the assembly of the North China Craton. Geology, 32, 229-232, doi: 10.1130/G20133.1. [Link]

Zhi, X., Y. Song, F. A. Frey, J. Feng, and M. Zhai, 1990: Geochemistry of Hannuoba basalts, eastern China: Constraints on the origin of continental alkalic and tholeiitic basalt. Chem. Geol., 88, 1-33, doi: 10.1016/000 9-2541(90)90101-C. [Link]

Zindler, A. and S. Hart, 1986: Chemical geodynamics. Ann. Rev. Earth Planet. Sci., 14, 493-571, doi: 10.1146/annurev.ea.14.050186.002425. [Link]

Zou, H., M. R. Reid, Y. Liu, Y. Yao, X. Xu, and Q. Fan, 2003: Constraints on the origin of historic potassic basalts from northeast China by U-Th disequilibrium data. Chem. Geol., 200, 189-201, doi: 10.1016/S00092541(03)00188-8. [Link] 